\title{
STAR-JET INTERACTIONS AND GAMMA-RAY OUTBURSTS FROM 3C454.3
}

\author{
D. V. Khangulyan ${ }^{1}$, M. V. Barkov ${ }^{2,3}$, V. Bosch-Ramon ${ }^{4}$, F. A. Aharonian ${ }^{5,2}$, And A. V. Dorodnitsyn ${ }^{6,7}$ \\ ${ }^{1}$ Institute of Space and Astronautical Science/JAXA, 3-1-1 Yoshinodai, Chuo-ku, Sagamihara, Kanagawa 252-5210, Japan \\ ${ }^{2}$ Max-Planck-Institut für Kernphysik, Saupfercheckweg 1, D-69117 Heidelberg, Germany \\ ${ }^{3}$ Space Research Institute RAS, 84/32 Profsoyuznaya Street, Moscow 117997, Russia \\ ${ }^{4}$ Departament d'Astronomia i Meteorologia, Institut de Ciències del Cosmos (ICC), Universitat de Barcelona (IEEC-UB), \\ Martí i Franquès 1, E-08028 Barcelona, Spain \\ ${ }^{5}$ Dublin Institute for Advanced Studies, 31 Fitzwilliam Place, Dublin 2, Ireland \\ ${ }^{6}$ Laboratory for High Energy Astrophysics, NASA Goddard Space Flight Center, Code 662, Greenbelt, MD 20771, USA \\ ${ }^{7}$ Department of Astronomy/CRESST, University of Maryland, College Park, MD 20742, USA \\ Received 2013 May 17; accepted 2013 July 7; published 2013 August 22
}

\begin{abstract}
We propose a model to explain the ultra-bright $\mathrm{GeV}$ gamma-ray flares observed from the blazar $3 \mathrm{C} 454.3$. The model is based on the concept of a relativistic jet interacting with compact gas condensations produced when a star (a red giant) crosses the jet close to the central black hole. The study includes an analytical treatment of the evolution of the envelope lost by the star within the jet, and calculations of the related high-energy radiation. The model readily explains the day-long that varies on timescales of hours, $\mathrm{GeV}$ gamma-ray flare from 3C454.3, observed during 2010 November on top of a plateau lasting weeks. In the proposed scenario, the plateau state is caused by a strong wind generated by the heating of the stellar atmosphere due to nonthermal particles accelerated at the jet-star interaction region. The flare itself could be produced by a few clouds of matter lost by the red giant after the initial impact of the jet. In the framework of the proposed scenario, the observations constrain the key model parameters of the source, including the mass of the central black hole: $M_{\mathrm{BH}} \simeq 10^{9} M_{\odot}$, the total jet power: $L_{\mathrm{j}} \simeq 10^{48} \mathrm{erg} \mathrm{s}^{-1}$, and the Doppler factor of the gamma-ray emitting clouds: $\delta \simeq 20$. Whereas we do not specify the particle acceleration mechanisms, the potential gamma-ray production processes are discussed and compared in the context of the proposed model. We argue that synchrotron radiation of protons has certain advantages compared to other radiation channels of directlyaccelerated electrons. An injected proton distribution $\propto E^{-1}$ or harder below the relevant energies would be favored to alleviate the tight energetic constraints and to avoid the violation of the observational low-energy constraints.
\end{abstract}

Key words: galaxies: jets - gamma rays: galaxies - radiation mechanisms: non-thermal

Online-only material: color figures

\section{INTRODUCTION}

$3 \mathrm{C} 454.3$ is a powerful flat-spectrum radio quasar located at a redshift $z_{\mathrm{rs}}=0.859$. This source is very bright in the $\mathrm{GeV}$ energy range; during strong flares, its apparent (isotropic) luminosity can reach $L_{\gamma} \gtrsim 10^{50} \mathrm{erg} \mathrm{s}^{-1}$ (e.g., Striani et al. 2010; Ackermann et al. 2010; Vercellone et al. 2011; Abdo et al. 2011). The mass of the central black hole (BH) in 3C454.3 is estimated to be in the range $M_{\mathrm{BH}} \approx(0.5-4) \times 10^{9} M_{\odot}$ (Gu et al. 2001; Bonnoli et al. 2011). This mass implies an Eddington luminosity $L_{\mathrm{Edd}} \approx(0.6-5) \times 10^{47} \mathrm{erg} \mathrm{s}^{-1}$, which is several orders of magnitude below $L_{\gamma}$. Although the large gap between $L_{\mathrm{Edd}}$ and $L_{\gamma}$ is naturally explained by relativistic Doppler boosting, estimates of the jet power during these flares appear, in any realistic scenario, close to or even larger than the Eddington luminosity (Bonnoli et al. 2011). Even though they are quite extreme, "super-Eddington" jets nevertheless cannot be excluded for accreting BHs with very high accretion rates but low radiation efficiencies. Although 3C454.3 is an exceptional case (see, for example, the discussion in Bonnoli et al. 2011), data from other objects provide additional evidence in favor of jets with super-Eddington mechanical powers (López-Corredoira \& Perucho 2012).

The $\mathrm{GeV}$ emission from 3C454.3 is highly erratic, with variability timescales as short as $3 \mathrm{hr}$, as reported, in particular, for the 2009 December flare (Ackermann et al. 2010). The most spectacular flare in terms of both variability and gamma-ray luminosity was observed in 2010 November by the AGILE and Fermi Large Area Telescope (Fermi/LAT; Vercellone et al. 2011; Abdo et al. 2011) telescopes. During this high state, with the most active phase lasting for five days, the apparent $\mathrm{GeV}$ luminosity achieved was $L_{\gamma} \approx 2 \times 10^{50} \mathrm{erg} \mathrm{s}^{-1}$. Around the flare maximum, the rising time was $t_{\mathrm{r}} \approx 4.5 \mathrm{hr}$ and the decay time was $t_{\mathrm{f}} \approx 15 \mathrm{hr}$. The detection of photons with energies up to $\approx 30 \mathrm{GeV}$, the short variability, and the contemporaneous X-ray flux constrain the Doppler boosting of the emitter to $\delta_{\min } \gtrsim 16$ to avoid severe internal $\gamma \gamma$-absorption in the X-ray radiation field (Abdo et al. 2011).

A remarkable feature of the gamma-ray emission from 3C454.3 is the so-called "plateau phase" revealed during the bright flare in 2010. This plateau phase is characterized by a long-term brightening of the source, a few weeks before the appearance of the main flare. Such plateau states have been observed by Fermi /LAT for three flares (e.g., Ackermann et al. 2010; Abdo et al. 2011), with the plateau emission being about an order of magnitude fainter than that of the main flare.

Remarkably, the rapid gamma-ray variability of $3 \mathrm{C} 454.3$ is accompanied by activity at lower energies. Simultaneous multiwavelength observations of the source during flares have revealed a strong correlation between optical and X-ray data. This correlation has been interpreted as evidence that the gamma-ray source is located upstream from the core of the $43 \mathrm{GHz}$ radio source, which is at a distance $z<$ few pc from the central BH (see, e.g., Jorstad et al. 2010, 2012; Wehrle et al. 2012). 
Over recent years, several works have attempted to explain the flaring gamma-ray activity of 3C454.3 within the framework of the standard synchrotron self-Compton (SSC) or external inverse-Compton (EIC) models (Katarzyński \& Ghisellini 2007; Ghisellini et al. 2007; Sikora et al. 2008; Bonnoli et al. 2011). In the SSC scenario, it is possible to reproduce the spectral energy distribution (SED) from optical wavelengths to gamma rays. In these models, most of the jet power is (unavoidably) carried by protons, and only a small fraction is contained in relativistic electrons and the magnetic field. The required proton-to-Poynting flux ratio, $L_{\mathrm{p}} / L_{\mathrm{B}} \sim 100$, is quite large. Such a configuration would be hard to reconcile, at least in the gamma-ray emitting region close to the central $\mathrm{BH}$, with an undisturbed jet that is launched by the Blandford-Znajek (Blandford \& Znajek 1977) type (BZ) process, in which the luminosity of the jet is dominated by Poynting flux and the jet consists of $e^{ \pm}$-pairs (see also Ruffini \& Wilson 1975; Lovelace 1976). In this regard, we should mention that recent relativistic magnetohydrodynamical (MHD) simulations of jet acceleration yield much less efficient conversions of magnetic energy into bulk motion kinetic energy; these calculations (Komissarov et al. 2007, 2009; Tchekhovskoy et al. 2010) predict a quite modest ratio $\left(L_{\mathrm{p}}+L_{e^{ \pm}}\right) / L_{\mathrm{B}} \lesssim 4$.

As stars and clumpy matter are expected to be present in the jet surroundings, they could be behind the powerful gamma-ray events in active galactic nuclei (AGNs; see, e.g., a discussion in Bosch-Ramon et al. 2012). In particular, a red giant (RG) can enter into the jet, lose its external layers, and in this way generate a strong perturbation inside the jet. This perturbation can accelerate particles and produce high-energy radiation.

The jet-RG interaction (JRGI) scenario has been invoked to explain the day-scale flares in the nearby non-blazar-type AGN M87 (Barkov et al. 2010; Blandford \& Königl 1979). The JRGI has also been applied to the TeV blazar PKS 2155-304 (Barkov et al. 2012a) to demonstrate that the jet-driven acceleration of debris from the $\mathrm{RG}$ atmosphere can explain the ultrafast variability of very high-energy (VHE) gamma-ray emission on timescales as short as $\tau \sim 200 \mathrm{~s}$. A distinct feature of the JRGI scenario is the high magnetization $\left(L_{\mathrm{B}} / L_{\mathrm{p}, \mathrm{e}} \gg 1\right)$ of the relativistic flows located at sub-parsec distances, where the gamma-ray production supposedly takes place. Although the strong magnetic field, $B \geqslant 10 \mathrm{G}$, dramatically reduces the efficiency of the inverse Compton scattering of electrons, it opens an alternative channel of gamma-ray production through synchrotron radiation of protons (Aharonian 2000; Mücke \& Protheroe 2001). The latter can be effectively realized only in the case of acceleration of protons to the highest possible energies, up to $10^{20} \mathrm{eV}$. Thus, the second (somewhat "hidden") requirement of this model is a very effective acceleration of protons with a rate close to the theoretical limit dictated by classical electrodynamics (Aharonian et al. 2002). Although somewhat speculative, this condition does agree with rather model-independent (derived from first principles) arguments that the relativistic jets in AGNs present the best candidate sites of production of the highest energy cosmic rays (Aharonian et al. 2002; Lemoine \& Waxman 2009). The large magnetic field and acceleration of protons to the highest possible energies, coupled with strong Doppler boosting in relativistic outflows, not only provide an extension of the gamma-ray spectra up to $\mathrm{TeV}$ energies, but also produce variability timescales as short as $1 \mathrm{hr}$ (Aharonian 2000).

It is interesting to also note that inverse Compton models can be accommodated, at least in principle, in the JRGI scenario.
Moreover, unlike SSC models of powerful blazars, in which the requirement of a very weak magnetic field (implying a deviation from the equipartition condition by orders of magnitude) generally is not addressed and explained, the JRGI scenario can offer a natural way for leptonic models to be effective assuming that the gamma-ray emission is produced through inverse Compton scattering in shocked clouds originally weakly magnetized (see Barkov et al. 2012b).

In this work, we show that the JRGI scenario is a viable mechanism for the explanation of the flares seen in 3C454.3. We also argue that within this model, the plateau state can form due to the interaction of the jet with a stellar wind excited by nonthermal (accelerated) particles that penetrate into the RG atmosphere. Given its extreme nature and importance, we will use the very powerful $\mathrm{GeV}$ flare of $3 \mathrm{C} 454.3$ that occurred in 2010 November as a template for our interpretations. We will show that this active period of $3 \mathrm{C} 454.3$, consisting of day-long flares with variabilities as short as a few hours on top of a plateau state lasting several weeks, can be explained by the interaction of a $\mathrm{RG}$ with the jet relatively close to the central $\mathrm{BH}$.

\section{THE STAR-JET INTERACTION SCENARIO}

The possibility that some exceptional flares in AGNs may originate from star-jet interactions has been proposed and discussed in our recent papers (Barkov et al. 2010, 2012a, $2012 b$ ). In this work, we explore the question whether the extremely bright and short gamma-ray flares detected by Fermi/ LAT from 3C454.3 in 2010 November can be explained by this model. It is important to note that these flares provide us with information of exceptional quality concerning both the temporal behavior of the source and its energy spectrum. In this regard, we should note that the operation of Fermi/LAT in scanning mode allows continuous monitoring of the source, so its temporal behavior, even during the pre- and post-flare epochs, can be studied without interruption. Before considering the case of $3 \mathrm{C} 454.3$, we discuss in this section the different implications of the JRGI scenario regarding the general temporal structure of the active phase in a blazar-type AGN.

When a star enters an AGN jet, some stellar material is expected to be released inside the jet flow, eventually forming a population of blobs that will be accelerated by the jet ram pressure. During this process, depending on the local magnetic fields, different types of shocks and plasma waves can be produced and propagate through both the jet material and the blobs. These waves may accelerate particles to relativistic energies. The interaction of these particles with the ambient radiation and magnetic fields would result in broadband nonthermal emission. Generally, the properties of this emission depend on the specifics of the acceleration and radiation mechanisms and the details of the target fields. However, in the case of extremely effective particle acceleration and radiation, i.e., when the corresponding acceleration and radiative cooling times are shorter than other timescales characterizing the dynamics of the system (a mandatory condition given the enormous luminosity of the emission), the lightcurve of the emission will be essentially determined by the jet/blob interaction model.

In the fast cooling regime, the proper intensity of the nonthermal emission, i.e., the intensity in the blob comoving reference frame, is proportional to the energy released at the jet-blob interface. This energy release can be characterized by a simple dynamical model, which describes the acceleration of the blob by the jet ram pressure. In this model, there are just a few relevant parameters that describe the basic properties of the jet 
and the blob: the jet ram pressure $\left(P_{\mathrm{j}}\right)$ and bulk Lorentz factor $\left(\Gamma_{\mathrm{j}}\right)$ and the blob mass $\left(M_{\mathrm{b}}\right)$ and radius $\left(r_{\mathrm{b}}\right.$; or, equivalently, its cross-section: $S_{\mathrm{b}}=\pi r_{\mathrm{b}}^{2}$; for details, see Barkov et al. 2012a). The time dependence of the intensity of the jet/blob interaction corrected for Doppler boosting can be treated as a first-order approximation for the radiation lightcurve.

There are several important stages in the JRGI scenario that may have an important impact on the observed lightcurve of the radiation: (1) first, the removal of the star's external layers; (2) the formation of a cloud from the removed stellar material, which under the jet impact suffers heating and expansion, and eventually fragments into a set of smaller blobs ${ }^{8}$ (see, e.g., Barkov et al. 2012a; Bosch-Ramon et al. 2012; Imshennik 1972); (3) the acceleration of the blobs in the direction of the jet flow to a Lorentz factor equal to or smaller than the jet Lorentz factor. The intensity of the nonthermal processes associated with the blob motion has a strong time dependence. At the start of the acceleration of the blob, its Lorentz factor is modest, yielding a small (but growing with time) boosting of the flux. At later stages, when the blob Lorentz factor approaches the Lorenz factor of the jet, the radiation intensity decreases because of the weakening of the jet-blob interaction. According to the results obtained in Barkov et al. (2012a) in the fast cooling limit, the maximum of the apparent luminosity, accounting for the Doppler boosting of the emission, occurs when the blob bulk Lorentz factor reaches $\Gamma_{\mathrm{b}} \sim 0.8 \Gamma_{\mathrm{j}}$.

During the penetration of the star into the jet, strong shocks are induced in the stellar atmosphere and a significant part of the envelope can be removed. Although the details of this process might be very complicated and depend on the radial density profile of the star and the structure of the jet outer boundary, here, for simplicity, we assume an instantaneous penetration. To a certain extent, this simplification is justified by the stellar proper motion, which is expected to be faster than the initially induced shock waves. Nevertheless, independent of the details of the star penetration, one can expect that when the star is fully within the jet, a dense cloud will be released. Later, as the cloud propagates through the jet, the star may still release an intense wind or a sequence of small clouds due to ablation of the stellar atmosphere by the jet. A sketch of the JRGI scenario is presented in Figure 1.

The mass of the cloud $\Delta M$ formed at the initial stage can be estimated by comparing the jet ram pressure,

$$
P_{0} \simeq \frac{L_{\mathrm{j}}}{c \pi \omega_{0}^{2}}
$$

with the gravitational force. Here, $\omega_{0}$ is the jet cross-section at the star crossing height. This gives the following estimate:

$$
\Delta M=\frac{\pi P_{0} R_{*}^{4}}{G M_{*}},
$$

where $M_{*}$ and $R_{*}$ are the RG mass and radius, respectively.

The absolute upper limit on the continuous mass-loss rate can be estimated by comparing the energy flux density of the jet $q_{0}=L_{\mathrm{j}} / \pi \omega_{0}^{2}$ and the gravitational force work density for removing matter from the stellar surface $q_{\mathrm{hd}}=\rho v G M_{*} / R_{*}=$ $\dot{M} G M_{*} /\left(\pi R_{*}^{3}\right)$. The maximum continuous mass ejection from

\footnotetext{
8 Throughout the paper, we will use the subscripts "c," "b," and " 0 " to characterize the parameters of the initially formed cloud, of the blob, and of the jet at the star crossing height, respectively.
}

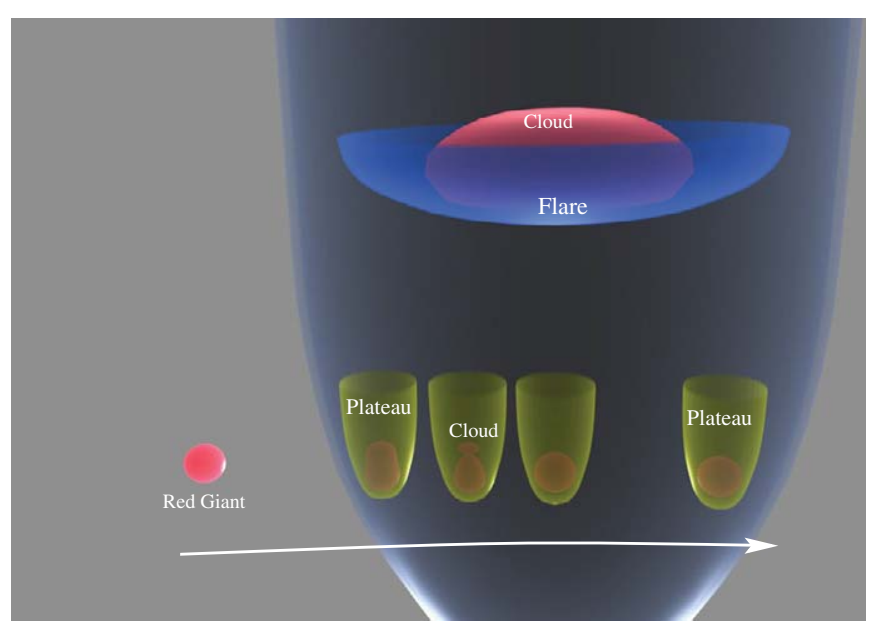

Figure 1. Sketch of the JRGI scenario, in which a star moving from left to right penetrates the jet. The star's external layers are shocked and carried away, and a cometary tail, the origin of the plateau emission, forms. The acceleration and expansion of the bigger clouds from the initially blown-up external layers of the star would lead to the main flare.

(A color version of this figure is available in the online journal.)

the star is

$$
\dot{M}=\frac{c \pi P_{0} R_{*}^{3}}{G M_{*}} .
$$

A more precise estimate of this rate requires a detailed study of the interaction process, which is beyond the scope of this paper. Nevertheless, in Section 3.2 we discuss whether a sufficiently strong stellar wind can be excited by the penetration of nonthermal (accelerated) particles.

Since the initial size of the expelled cloud should be comparable to the size of the star, it is possible to estimate the cloud expansion time as $t_{\exp } \propto 2 R_{*} / c_{\mathrm{S}}$, where $c_{\mathrm{S}}$ is the sound speed of the shocked material: $c_{\mathrm{s}} \approx\left[\left(4 \pi R_{*}^{3} / 3\right) \gamma_{\mathrm{g}} P_{0} / M_{\mathrm{c}}\right]^{1 / 2}$. The cloud expansion time is

$$
t_{\exp } \approx A_{\exp }\left(\frac{M_{\mathrm{c}}}{\gamma_{\mathrm{g}} R_{*} P_{0}}\right)^{1 / 2},
$$

where $\gamma_{\mathrm{g}}=4 / 3$ is the plasma adiabatic coefficient and $A_{\text {exp }}$ is a constant of about a few (Gregori et al. 2000; Nakamura et al. 2006; Pittard et al. 2010; Bosch-Ramon et al. 2012). According to the relativistic hydrodynamic (RHD) simulation by BoschRamon et al. (2012), a value of 1.5 can be adopted for $A_{\text {exp }}$.

The blob acceleration occurs on a timescale of (see, e.g., Barkov et al. 2012a):

$$
t_{\mathrm{acc}} \approx\left\{\begin{array}{cl}
\frac{z_{0}}{c} & \text { if } D<1 \\
\frac{z_{0}}{c} \frac{1}{D} & \text { if } D>1 .
\end{array}\right.
$$

The $D$-parameter that will be often used in the paper has a simple meaning. It is the dimensionless inverse mass of the blob:

$$
D \equiv \frac{P_{0} \pi r_{\mathrm{b}}^{2} z_{0}}{4 c^{2} M_{\mathrm{b}} \Gamma_{0}^{3}} .
$$

The above timescale corresponds to the blob acceleration in the laboratory reference frame. However, since the blob gets accelerated toward the observer, the emission delay, as seen by the observer, should be approximately corrected by a factor of $1 /\left(2 \Gamma_{0}^{2}\right)$. Thus, the observed peak of the emission should be delayed by a time interval of

$$
\Delta t=t_{\exp }+t_{\mathrm{acc}} /\left(2 \Gamma_{0}^{2}\right) .
$$


There are some uncertainties in this equation. In particular, for $D<1$, the blobs can travel a distance comparable to $z$ and the jet properties at the dominant emission blob location may differ significantly from those at the blob formation site. Thus, the detailed evolution of the emitter can only be addressed properly through numerical modeling. However, despite the adopted simple approach, some important conclusions can be derived. Namely, given the dependence of the two components on the right-hand side of Equation (7) on the stellar material mass ( $M_{\mathrm{c}}^{1 / 2}$ and $M_{\mathrm{b}}$, respectively), the radiation associated with the heavy cloud expelled first will be delayed with respect to the emission produced by lighter clouds formed later.

The emission produced by lighter clouds allows an estimation of the time required for the star to cross the jet. Once the star enters into the jet, the process of jet-star interaction should proceed steadily, with the production of these lighter clouds being roughly constant on average. Thus, the whole duration of the light cloud-associated emission, if observed, can be taken as a direct measurement of the jet crossing time $t_{0} \approx 2 \omega_{0} / V_{\text {orb }}$, where $V_{\text {orb }} \lesssim \sqrt{2 G M_{\mathrm{BH}} / z_{0}}$ is the stellar velocity. This yields the following relation:

$$
t_{0} \gtrsim \sqrt{2} \frac{\omega_{0} z_{0}^{1 / 2}}{c r_{\mathrm{g}}^{1 / 2}}
$$

where $r_{\mathrm{g}}$ is the $\mathrm{BH}$ gravitational radius. Adopting the paradigm of magnetically accelerated jets (see Appendix A, Equations (A2)-(A4)), it is possible to derive a very simple expression for this timescale:

$$
t_{0} \gtrsim 2^{3 / 2} z_{0} / c
$$

In this way, the duration of the jet-star interaction is determined by the interaction distance from the central $\mathrm{BH}$.

The physical properties of the emitting blobs determine the available energetics and the maximum flux reachable in the considered scenario. In the blob comoving frame, the jet-star interface energy flux is defined by

$$
q_{\mathrm{b}}=\left(\frac{1}{\Gamma_{\mathrm{b}}^{2}}-\frac{\Gamma_{\mathrm{b}}^{2}}{\Gamma_{\mathrm{j}}^{4}}\right) \frac{c P_{\mathrm{j}}}{4} .
$$

Assuming a fixed efficiency $\xi$ in the blob reference frame for the transfer of jet power to nonthermal gamma rays (where $\xi \ll 1$ ), and correcting for Doppler boosting, one can estimate the luminosity of a blob:

$$
L_{\gamma}=4 \xi c F_{\mathrm{e}} P_{0} \Gamma_{0}^{2} \pi r_{\mathrm{b}}^{2},
$$

where the correction function $F_{\mathrm{e}}$ depends on time, or, equivalently, on the blob location in the jet (for a mathematical definition of this function, see Appendix B, Equation (B8)). We note that the structure of the jet, i.e., the dependence of the jet Lorentz factor on $z$, determines the actual dependence of $F_{\mathrm{e}}$ on $z$ (also see Appendix B for details).

The maximum value of $F_{\mathrm{e}}$ monotonically depends on the $D$-parameter, approaching a value of 0.4 if $D \gtrsim 1$ and a value of $\sim 0.1$ for $D=0.1$. This relatively weak dependence allows us to derive the maximum blob luminosity. Also, it is possible to obtain an estimate of the total energy emitted by a blob or an ensemble of sub-blobs as a result of the fragmentation of the original cloud $\left(M_{\mathrm{c}}=\sum M_{\mathrm{b}}\right)$ :

$$
E_{\gamma} \simeq 8 \xi \bar{F}_{\mathrm{e}} M_{\mathrm{b} / \mathrm{c}} c^{2} \Gamma_{0}^{3}
$$

which accounts for the total energy transferred by the jet to a blob during the acceleration process, $M_{\mathrm{b}} c^{2} \Gamma_{0}$, and for the anisotropy of the emission due to relativistic effects represented by the factor $\Gamma_{0}^{2}$ (see Appendix B, in particular Equation (B9), for details).

\section{THE 2010 NOVEMBER FLARE}

\subsection{General Structure of the Active Phase}

The total apparent energy of the $\mathrm{GeV}$ gamma-ray radiation detected during the flare observed from 3C454.3 in 2010 November was about $E_{\mathrm{tot}} \approx L_{\gamma} \Delta t /\left(1+z_{\mathrm{rs}}\right) \approx 2.3 \times 10^{55} \mathrm{erg}$. The exceptionally high flux during this period allows the derivation of a very detailed lightcurve, as seen from Figure 1 in Abdo et al. (2011). The nonthermal activity lasted for $t_{\text {full }} \sim$ 80 days. The onset of the activity period was characterized by a plateau stage. During the first $t_{\mathrm{pl}} \sim 13$ days, a rather steady flux was detected, with an apparent luminosity $L_{\mathrm{pl}} \approx 10^{49} \mathrm{erg} \mathrm{s}^{-1}$. The plateau stage was followed by an exceptionally bright flare, the total duration of which was $t_{\mathrm{fl}} \sim 5$ days, with a rise time of $t_{\mathrm{r}} \sim 4.5 \mathrm{hr}$. The maximum flux reached was $7 \times 10^{-5}$ photons $\mathrm{cm}^{2} \mathrm{~s}^{-1}$, which corresponds to a luminosity of $L_{\gamma} \simeq 2 \times 10^{50} \mathrm{erg} \mathrm{s}^{-1}$. The final stage of the flare phase was characterized by variable emission with a flux approximately a factor of $\sim 5$ weaker than the main flare, but still a factor of $\sim 2$ above the plateau level.

The observed luminosity of the plateau phase allows us to determine a lower limit on the star mass-loss rate by differentiating Equation (12):

$$
\dot{M}_{*} \approx 10^{23} L_{\mathrm{pl}, 49} \xi^{-1} \Gamma_{0,1.5}^{-3} \mathrm{~g} \mathrm{~s}^{-1},
$$

where $L_{\mathrm{pl}, 49}=L_{\mathrm{pl}} / 10^{49} \mathrm{erg} \mathrm{s}^{-1}$. Comparing this requirement to Equation (3), it is easy to see that for typical parameters of RG stars, Equation (13) represents a modest fraction (a few percent) of the maximum possible mass-loss rate. In Section 3.2, we will return to this issue.

To evaluate the feasibility of the JRGI scenario for the 3C454.3 main flare, it is necessary to check whether the flux, the total energy release, and the flare delay with respect to the onset of the plateau are well described by Equations (7), (11), and (12) for a reasonable choice of jet/star properties. For example, Equation (11) can be rewritten as

$$
P_{0}=8 \times 10^{3} F_{\mathrm{e}, \max }^{-1} L_{\gamma, 50} \Gamma_{0,1.5}^{-2} S_{\mathrm{b}, 32}^{-1} \xi^{-1} \mathrm{erg} \mathrm{cm}^{-3},
$$

where $L_{\gamma, 50}$ is the peak luminosity of the flare normalized to $10^{50} \mathrm{erg} \mathrm{s}^{-1}$ and $S_{\mathrm{b}, 32}=\pi r_{\mathrm{b}}^{2} / 10^{32} \mathrm{~cm}^{2}$ is the blob cross-section.

A total energy budget of the flare of $\sim 2 \times 10^{55} \mathrm{erg}$ is feasible, according to Equation (12), if

$$
M_{\mathrm{c}, 30} \Gamma_{0,1.5}^{3} \approx \frac{0.04 E_{\gamma, 55}}{\xi \bar{F}_{\mathrm{e}}} \approx \frac{0.1}{\xi \bar{F}_{\mathrm{e}}},
$$

where $M_{\mathrm{c}, 30}=M_{\mathrm{c}} / 10^{30} \mathrm{~g}$ is the mass of the blown-up RG envelope (i.e., the initially formed cloud). This requirement appears to be very close to that provided by Equation (2), where the jet ram pressure is now substituted using Equation (14):

$$
M_{\mathrm{c}, \max } \approx \frac{5 \times 10^{29}}{F_{\mathrm{e}, \max }} R_{*, 2}^{4} M_{*, 0}^{-1} L_{\gamma, 50} \Gamma_{0,1.5}^{-2} S_{\mathrm{b}, 32}^{-1} \mathrm{~g},
$$

where $R_{*, 2}=R_{*} / 10^{2} R_{\odot}$ and $M_{*, 0}=M_{*} / M_{\odot}$, respectively. 
The second term in Equation (7) is expected to be short compared to the duration of the plateau phase, even for $D \sim 0.1$, and thus the duration of the initial plateau phase constrains the expansion time (see Equation (4)):

$$
\begin{aligned}
t_{\exp } \approx & 5.4 \times 10^{6} F_{\mathrm{e}, \max }^{1 / 2} \xi^{1 / 2} \\
& \times M_{\mathrm{c}, 30}^{1 / 2} R_{*, 2}^{-1 / 2} L_{\gamma, 50}^{-1 / 2} \Gamma_{0,1.5} S_{\mathrm{b}, 32}^{1 / 2} \mathrm{~s} .
\end{aligned}
$$

Abdo et al. (2011) found that the emission of the main flare consisted of five components (see Figure 2 in that work): a nearly steady contribution, like a smooth continuation of the plateau emission, and four sub-flares of similar duration and energetics. Although the uniqueness of such a fit is not statistically assessed, it looks like a good empirical description of the data. In the framework of the JRGI scenario, such a description is very natural. The steady component would be attributed to light clouds, continuously ejected by the star, and the four subflares would correspond to much heavier blobs formed out of the blown-up stellar envelop during the initial stage. On the other hand, the decomposition of the main flare in four subflares implies a strict limitation on the variability timescale. The flare rise/decay timescales should be longer than the blob light crossing time corrected for Doppler boosting. Since the shortest variability scale was $\sim 5 \mathrm{hr} /\left(1+z_{\mathrm{rs}}\right) \sim 10^{4} \mathrm{~s}$, the maximum possible size of the emitting blobs can be estimated as:

$$
r_{\mathrm{b}} \approx 10^{16} \Gamma_{0,1.5} \mathrm{~cm}
$$

If the jet is magnetically driven, this size constraint can be expressed through the mass of the central BH (see Equation (A2)):

$$
\frac{r_{\mathrm{b}}}{\omega}<0.5 M_{\mathrm{BH}, 9}^{-1},
$$

which is restrictive only in the case of $M_{\mathrm{BH}, 9} \gg 1$. For $M_{\mathrm{BH}, 9} \lesssim 1$, the blobs can cover the entire jet without violating the causality constraint.

In summary, if the flare detected with Fermi/LAT was produced by an RG entering into the jet, the jet properties should satisfy Equations (14), (15), and (17), which correspond to the restrictions imposed by the flux level, total energy release, and the duration of the plateau stage, respectively. Interestingly, this set of equations allows the derivation of a unique solution, which can constrain all the key parameters through the value of the $D$-parameter:

$$
\begin{gathered}
P_{0}=3 \times 10^{6} \frac{F_{\mathrm{e}, \max }^{1.5} D^{1.5}}{\bar{F}_{\mathrm{e}}^{2.5} \xi z_{0,17}^{1.5}} \mathrm{erg} \mathrm{cm}^{-3}, \\
M_{\mathrm{c}}=4 M_{\mathrm{b}}=5 \times 10^{30} \frac{F_{\mathrm{e}, \max }^{1.5} D^{1.5}}{\bar{F}_{\mathrm{e}}^{2.5} \xi z_{0,17}^{1.5}} \mathrm{~g}, \\
\Gamma_{0}=8\left(\frac{\bar{F}_{\mathrm{e}, 0,17} z_{0}}{F_{\mathrm{e}, \max } D}\right)^{0.5},
\end{gathered}
$$

and

$$
S_{\mathrm{b}}=8 \times 10^{30} \frac{z_{0,17}^{0.5} \bar{F}_{\mathrm{e}}^{1.5}}{F_{\mathrm{e}, \max }^{1.5} D^{0.5}} \mathrm{~cm}^{2} .
$$

The value of the ram pressure determined by Equation (20) can be compared to the value achievable in a magnetically driven jet:

$$
P_{\text {mdj }}=2 \times 10^{4} z_{0,17}^{-1} \frac{L_{\mathrm{j}}}{L_{\text {Edd }}} \operatorname{erg~cm}^{-3},
$$

where $z$ is the distance from the central BH. It can be seen from a comparison of Equation (20) and Equation (24) that the solution found implies a jet with a luminosity exceeding the Eddington limit by a factor of $>10$. This requirement is also consistent with the lower limit on the jet luminosity

$$
L_{\mathrm{j}}>c S_{\mathrm{b}} P_{0}=8 \times 10^{47} z_{0,17}^{-1} \xi^{-1} \frac{D}{\bar{F}_{\mathrm{e}}} \operatorname{erg~s}^{-1},
$$

which exceeds the Eddington limit for the mass of the central $\mathrm{BH} M_{\mathrm{BH}} \sim 5 \times 10^{8} M_{\odot}$ (see Bonnoli et al. 2011 and references therein). To assess the feasibility of such a strongly superEddington jet is beyond the scope of this paper, although we note that López-Corredoira \& Perucho (2012) presented observational evidence indicating that such jets may not be uncommon. We also note that the requirement of a high luminosity is less severe in the case of small $D$ values and that in this limit one can derive a constraint on the central $\mathrm{BH}$ mass by combining Equations (8) and (23):

$$
M_{\mathrm{BH}}>7 \times 10^{8} M_{\odot} z_{0,17}^{3 / 2} .
$$

The coherent picture emerging from the jet properties derived above suggests that the JRGI scenario can be responsible for the flare detected from 3C454.3 for a solution of the problem with a reasonable set of model parameters. This solution is designed to satisfy the requirements for (1) the total energy; (2) the peak luminosity; and (3) the duration of the plateau phase. Therefore, some additional observational tests are required to prove the feasibility of the suggested scenario. To address this issue, we discuss in Section 4 the feasibility of different radiation mechanisms to explain the observations under the inferred emitter conditions. Also, in Section 3.2, we explore whether the stellar wind induced by the RG-jet interaction can be responsible for the flux level detected during the plateau stage. Recall that, as already shown, the required mass-loss rate is well within the limitations imposed by Equation (3).

Finally, the flare raise time, which is related to the blob acceleration timescale (see Equation (5)), can be calculated for the obtained solution. Interestingly, in the limit of small $D$ values, this timescale appears to be independent of $D$, the only remaining free parameter, and matches closely the detected raise time of $t_{\mathrm{r}} \sim 4.5 \mathrm{hr}$ :

$$
t_{\mathrm{acc}} /\left(2 \Gamma_{\mathrm{b}}^{2}\right) \simeq 5 \mathrm{hr} .
$$

This agreement can be treated as a cross-check that shows the feasibility of the proposed scenario.

\subsection{The Stellar Wind as the Origin of the Plateau Emission}

When the star penetrates the jet, strong perturbations are generated in both the jet structure and the star's external layers. Strong shocks and other processes of energy dissipation take place in the impacted jet and stellar material, generating conditions under which nonthermal particles can be accelerated. Here, we will not specify the acceleration mechanism, which, depending on the acceleration region, may be Fermi I, stochastic acceleration, or magnetic reconnection. The accelerated particles can either be advected away, while radiating, from the perturbed jet region, or penetrate into the stellar atmosphere and thermalize heating the ambient plasma. The heating can induce a strong wind that will significantly enhance the stellar mass-loss rate. A similarly enhanced mass loss can occur as well through Compton-heated winds in high-mass X-ray binaries and AGNs 
due to heating by X-rays from an accretion disk (e.g., Basko \& Sunyaev 1973; Dorodnitsyn et al. 2008a, 2008b), although for the mass-loss rate required to explain the plateau phase (see Equation (13)), the accretion disk X-ray flux seems to be too small. On the other hand, the nonthermal particles produced in the star-jet interaction region can be enough to heat the stellar atmosphere. Since even nonthermal particles with low energy can effectively heat the atmosphere, the nonthermal emission may not be detectable during the heating process. The heating energy flux can be estimated as

$$
F_{\mathrm{nt}}=\chi c P_{0}=3 \times 10^{16} \chi P_{0,6} \mathrm{erg} \mathrm{cm}^{-2} \mathrm{~s}^{-1},
$$

where $P_{0,6}=P_{0} /\left(10^{6} \mathrm{erg} \mathrm{cm}^{-3}\right)$ is the normalized jet ram pressure and $\chi=X_{\mathrm{a}} Y_{\text {dif }}$ is the nonthermal particle-to-jet power ratio within the stellar atmosphere: a combination of the fraction of the jet power channeled into nonthermal particles $X_{\mathrm{a}}$ and the fraction of the nonthermal particles diffusing into the $R G$ atmosphere $Y_{\text {dif }}$. In the case of a jet interacting with a heavy obstacle, the value of $X_{\mathrm{a}}$ could potentially be close to 1 . The entrainment of particles into the RG atmosphere could also be high due to a strongly asymmetric diffusion process: whereas the strong magnetic field in the jet may prevent particles from crossing back, the weaker magnetic field in the other side would stimulate particles to diffuse deep into the stellar atmosphere. For all this, the effective value of $\chi$ may be of the order of 1 .

The mass flux of a stellar wind excited by nonthermal particles of flux $F_{\text {nt }}$ can be estimated as:

$$
\begin{aligned}
\mu & =10^{-12} \alpha_{-12} \frac{F_{\mathrm{nt}} R_{*}^{1 / 2}}{\left(G M_{*}\right)^{1 / 2}} \\
& =7 \times 10^{-3} \alpha_{-12} R_{*, 2}^{1 / 2} M_{*, 0}^{-1 / 2} \chi P_{0,6} \mathrm{~g} \mathrm{~s}^{-1} \mathrm{~cm}^{-2},
\end{aligned}
$$

where $\alpha$ is related to the properties of the plasma heating and cooling. In the case of X-ray heating, one finds that $\alpha \sim 0.03 / c$ or $\alpha_{-12} \sim 1$ (i.e., Basko et al. 1977). However, for nonthermalparticle heating, the efficiency of transferring jet energy into the stellar wind can be higher because these particles can penetrate deeper into the stellar wind. Thus, in this case, $\alpha_{-12}$ can significantly exceed 1 . The induced mass-loss rate therefore will be $\dot{M}_{\mathrm{w}}=\pi \mu R_{*}^{2}$, or

$$
\dot{M} \approx 10^{24} \alpha_{-12} R_{*, 2}^{5 / 2} M_{*, 0}^{-1 / 2} \chi P_{0,6} \mathrm{~g} \mathrm{~s}^{-1} .
$$

To explain the plateau emission by the interaction of this induced stellar wind with the jet, the mass flux from Equation (30) should be greater than the one from Equation (13). Adopting the typical parameters of the 2010 November flare, this requirement can be satisfied if the following condition holds (the function $\bar{F}_{\text {e }}$ here characterizes the main flare episode):

$$
\alpha_{-12} \chi \gtrsim 2 \bar{F}_{\mathrm{e}} R_{*, 2}^{-5 / 2} M_{0, *}^{1 / 2} .
$$

Formally, for the adopted RG normalization parameters, the required heating efficiency should be rather high: $\chi \approx 0.7$ (accounting for $\bar{F}_{\mathrm{e}}<0.3$, as found for conical jets-see Appendix B for details). However, a significantly lower heating efficiency can be sufficient to fulfill Equation (31) under certain circumstances; $\chi$ could be easily reduced if $D \ll 1\left(\bar{F}_{\mathrm{e}} \approx D\right.$ in this regime), or assuming that nonthermal particles contribute strongly to the pressure of the stellar atmosphere $\left(\alpha_{-12} \gg 1\right)$, or for a $\mathrm{RG}$ radius exceeding the fiducial value $R_{*}=100 R_{\odot}$. For instance, to reduce $\chi$ to 0.1 , one may adopt either $R_{*}=200 R_{\odot}$ or $\alpha_{-12} \approx 5$ and $R_{*}=100 R_{\odot}$ or $D \approx 0.05$. A combination of all these factors could further decrease the required heating efficiency to lower values. Therefore, an induced stellar wind seems to provide a feasible way to generate the plateau emission component.

\section{RADIATION MECHANISMS}

\subsection{General Comments}

In this section, we discuss the feasibility of different radiation mechanisms for the production site conditions inferred in the previous section (see Equations (20)-(23)). The efficiency of the radiation channels is determined by the presence of nonthermal particles with the required energy and targets. Several types of target fields are related to efficient highenergy processes: matter in the case of the proton-proton or Bremsstrahlung mechanisms, a magnetic field in case of synchrotron radiation, and photons for inverse Compton and photomeson emission. The obtained solution for the properties of the jet, plus observational constraints, allow two of these targets to be properly characterized.

Since the mass and size of the blobs are estimated, one can derive the matter density in the production regions:

$$
n_{\mathrm{b}} \sim 10^{7} \mathrm{~cm}^{-3}
$$

This density estimate allows one to discard the proton-proton channel, since the expected cooling time, $t_{\mathrm{pp}} \approx 10^{15} / \mathrm{n} \mathrm{s} \sim$ $10^{9} \mathrm{~s}$, is too long. ${ }^{9}$ This conclusion also excludes the Bremsstrahlung channel, which has a similar cooling time.

The derived ram pressure of the jet allows the derivation of an upper limit on the magnetic field strength:

$$
B_{\mathrm{j}}<6 \times 10^{3} \frac{F_{\mathrm{e}, \max }^{3 / 4} D^{3 / 4}}{F_{\mathrm{e}}^{5 / 4} \xi^{1 / 2} z_{0,17}^{3 / 4}} \mathrm{G} .
$$

In fact, from equipartition arguments, the actual strength of the magnetic field should be close to that value; i.e., one may take $x \sim 1$ as the fiducial value for the fraction of the jet luminosity carried in electromagnetic form. Also, accounting for the relation $F_{\mathrm{e}, \max }^{3 / 4} D^{3 / 4} / \bar{F}_{\mathrm{e}}^{5 / 4} \geqslant 0.7$, valid in the range of feasible values of the $D$-parameter for the conical jet model, one can derive a lower limit on the jet magnetic field of

$$
B_{\mathrm{j}}>3 \times 10^{3} \xi^{-1 / 2} \varkappa^{1 / 2} z_{0,17}^{-3 / 4} \mathrm{G} .
$$

More detailed calculations of this value are presented in Figure 2. In this figure, we also show the value of the magnetic field in the comoving system $\left(B_{\mathrm{j}}^{\prime}\right)$, which was calculated using the jet Lorenz factor from Equation (22) as a function of the value of $D$. It can be seen that for the $D$-range of interest, there is a lower limit on the magnetic field strength:

$$
\begin{aligned}
B_{\mathrm{j}}^{\prime}=B_{\mathrm{j}} \Gamma_{\mathrm{b}}^{-1} & >B_{\mathrm{j}} \Gamma_{0}^{-1} \\
& >200 \xi^{-1 / 2} \varkappa^{1 / 2} z_{0,17}^{-5 / 4} \mathrm{G} .
\end{aligned}
$$

Regarding the density of the target photon field, it cannot be constrained in general. However, in the specific case of the

\footnotetext{
9 We note, however, that this limitation does not apply in the case of "off-axis" AGNs, since then there are no constraints related to the requirement of blob acceleration. In particular, proton-proton interactions were shown to be a feasible channel for the interpretation of the $\mathrm{TeV}$ emission detected from M87 (Barkov et al. 2010, 2012b).
} 

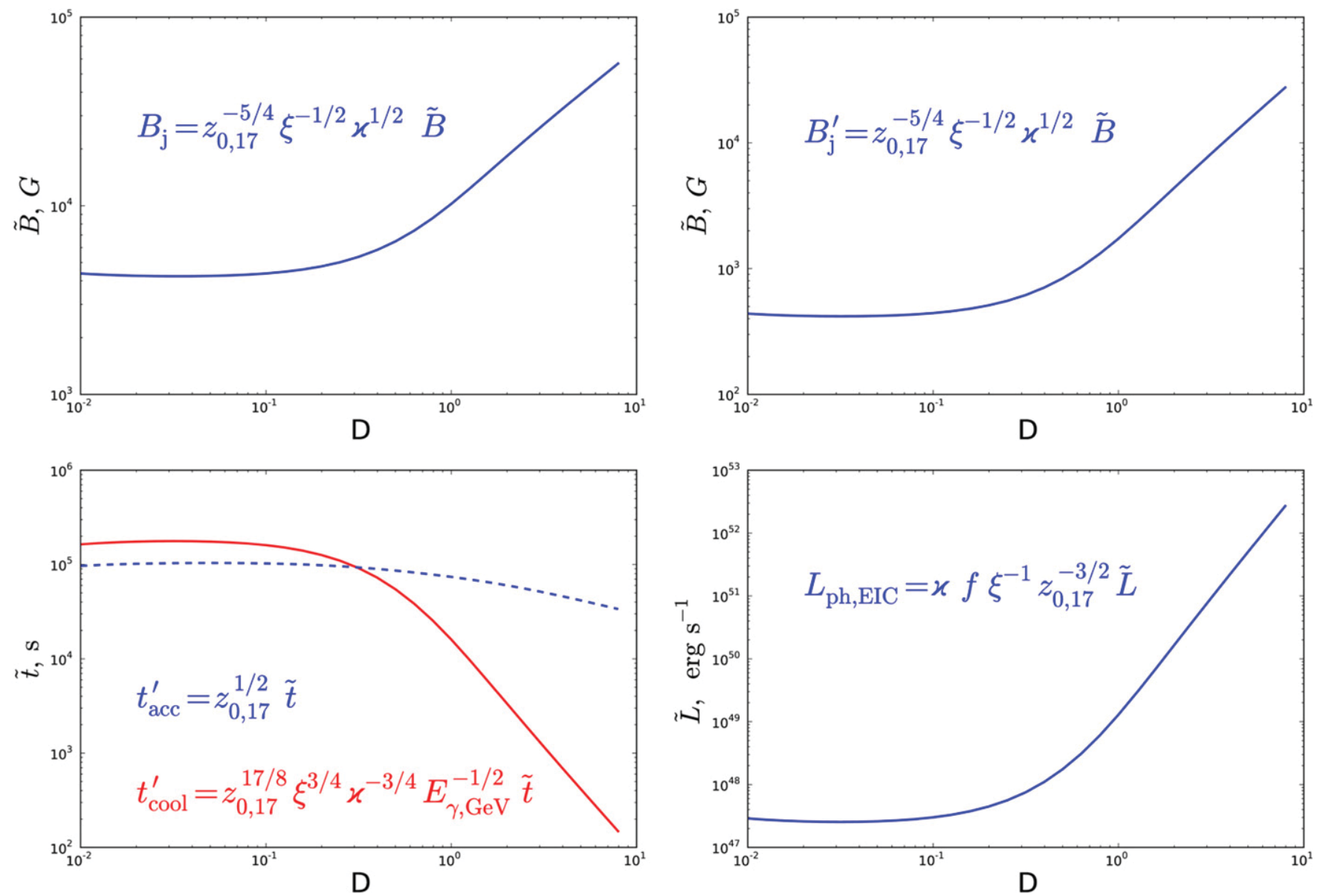

Figure 2. Numerical calculations of the parameters related to radiation processes for the case of a conic jet vs. the value of $D$. Top left panel: jet magnetic field; top right panel: jet magnetic field in the comoving frame; bottom left panel: proton synchrotron cooling time (red solid line) and blob acceleration time (blue dashed line), both in the comoving frame; bottom right panel: the registered luminosity of the target photon field for the EIC scenario.

(A color version of this figure is available in the online journal.)

SSC scenario, it is possible to apply certain limitations. Namely, the high-energy component detected by Fermi/LAT exceeds by two orders of magnitude $(f \approx 100)$ the flux detected at other wavelengths. This finding implies that the energy density of the target field should exceed the energy density of the magnetic field by the same factor: $w_{\mathrm{ph}}^{\prime}=f w_{\mathrm{B}}^{\prime}$ (these are comoving reference frame values). The luminosity of such a field can be estimated as

$$
\begin{aligned}
L_{\text {target }} & \sim 4 \pi r_{\mathrm{b}}^{2} c w_{\mathrm{ph}}^{\prime} \Gamma_{\mathrm{b}}^{4} \sim 4 \pi r_{\mathrm{b}}^{2} c f w_{\mathrm{B}}^{\prime} \Gamma_{\mathrm{b}}^{4} \\
& \sim 4 \pi r_{\mathrm{b}}^{2} c f \varkappa P_{0} \Gamma_{\mathrm{b}}^{2} \sim F_{\mathrm{e}}^{-1}\left(\frac{\Gamma_{\mathrm{b}}}{\Gamma_{0}}\right)^{2} \xi^{-1} f \varkappa L_{\gamma} .
\end{aligned}
$$

Since $f L_{\text {target }}<L_{\gamma}$ and $F_{\mathrm{e}} \simeq 0.5\left(\Gamma_{\mathrm{b}} / \Gamma_{0}\right)^{2}$, one obtains

$$
\varkappa f^{2}<0.5 \xi
$$

Thus, the SSC scenario can be realized only in a specific region of the jet, where the magnetic field is significantly lower than the characteristic one (i.e., $\varkappa<10^{-4}$ ). The blobs themselves may have a magnetic field quite different from the field in the jet, and therefore may serve as good sites for SSC. However, this assumption involves further complexity, e.g., regarding internal shock acceleration within the blobs, and is therefore deferred to future studies. Also, we note that the radiation efficiency of photomeson production is low (e.g., see the discussion in
Aharonian et al. 2008). If photomeson production were the radiation mechanism, it would imply a very low value of $\xi$ and an uncomfortably high power of the jet.

We focus now on the following radiation mechanisms: electron and proton synchrotron and EIC. The analysis of the synchrotron channel is straightforward, since the density of the target is determined. Indeed, to produce a gamma ray of energy $E_{\gamma, \mathrm{GeV}}=E_{\gamma} / 1 \mathrm{GeV}$, either an electron or proton of energy

$$
E_{\mathrm{e} / \mathrm{p}}=2\left(\frac{m_{e / p}}{m_{e}}\right)^{3 / 2} \xi^{1 / 4} \varkappa^{-1 / 4} z_{0,17}^{3 / 8} E_{\gamma, \mathrm{GeV}}^{1 / 2} \mathrm{TeV}
$$

is required. We note that the weak $D$-dependence in these equations is neglected for simplicity.

To obtain such a high-energy particle, a few conditions should be satisfied. The most critical of the conditions are the Hillas criterion and the efficiency of the acceleration process. The Hillas criterion requires that the gyroradius of the highest energy particles is smaller than the size of the acceleration region. Since the acceleration region size is determined by the blob crosssection, one can derive the following requirements:

$$
\xi^{3 / 4} \varkappa^{-3 / 4} z_{0,17}^{11 / 8} E_{\gamma, \mathrm{GeV}}^{1 / 2}<10^{8}\left(\frac{m_{\mathrm{e}}}{m_{\mathrm{e} / \mathrm{p}}}\right)^{3 / 2} .
$$

This estimate allows one to illustrate that for the derived jet parameters, the Hillas criterion appears to constrain the 
acceleration of particles in neither the lepton nor the proton synchrotron scenarios.

Regarding the efficiency of the acceleration process, it is convenient to present the nonthermal particle acceleration time in a form independent of the specific acceleration mechanism:

$$
t_{\mathrm{par}}=\frac{\eta R_{\mathrm{gy}}}{c}=\frac{\eta E_{\mathrm{e} / \mathrm{p}}}{e B_{\mathrm{j}}^{\prime} c},
$$

where $E_{\mathrm{e} / \mathrm{p}}$ is the energy of particle, $\eta \geqslant 1$ is a dimensionless parameter characterizing the acceleration process, and $R_{\mathrm{gy}}$ is the particle gyroradius. This timescale should be compared to the dominant cooling time (synchrotron) to determine the highest achievable energy:

$$
t_{\mathrm{sy}}=\frac{9 m_{e / p}^{4} c^{7}}{4 e^{4} E_{e / p} B_{\mathrm{j}}^{\prime 2}}>t_{\mathrm{par}} .
$$

Accounting for the energy of the emitted gamma rays, $E_{\gamma}$, one can obtain an upper limit on the acceleration time through a constraint on the value of $\eta$ :

$$
\eta<5 \times 10^{3} \Gamma_{\mathrm{b}, 1}\left(\frac{E_{\gamma}}{m_{\mathrm{e} / \mathrm{p}} c^{2}}\right)^{-1},
$$

where $\Gamma_{\mathrm{b}, 1}=\Gamma_{\mathrm{b}} / 10$ is the blob Lorentz factor. Since the redshift corrected energy of the spectral break appears to be close to 2 $\mathrm{GeV}$, the leptonic scenario can be realized only if $\Gamma_{\mathrm{b}}>10 \eta$. In the case of proton-synchrotron models, this constraint gets significantly relaxed, yielding $\eta<200 \Gamma_{\mathrm{b}}$. However, given the relatively small values of the obtained Lorentz factors (see Equation (22)), the derived limits on the acceleration efficiency are very tight, especially for the leptonic case $(\eta \rightarrow 1)$. The feasibility of such a scenario should be addressed with a detailed acceleration model, which remains beyond the scope of the present paper. We note that this limitation can be relaxed by adopting a scenario with a highly turbulent magnetic field (see more details in Kelner et al. 2013).

Since the proton synchrotron scenario fulfills the basic requirements for the production mechanism, it is worthwhile to check whether one can reproduce the observed basic spectral properties. Fermi/LAT reported a broken power law spectrum with a nearly constant break energy. The change of the power law index varies between 0.5 and 1 . Whereas index changes of 0.5 , thought to be cooling breaks, are typical in synchrotron scenarios, other values for the power law index change may be hard to explain through cooling. On the other hand, one can attribute this spectral feature to absorption (as suggested for this source by Stern \& Poutanen 2011).

The extrapolation of the synchrotron spectrum below the Fermi/LAT range requires a harder slope than at $\mathrm{GeV}$ energies, $n_{v} \propto v^{-1.5}$ or harder, to not overpredict the X-ray flux found by Swift. This harder slope implies a break in the photon distribution around energies of 10-100 MeV. Such a harder spectrum could be associated with the uncooled part of the particle distribution. The synchrotron cooling time of protons in the comoving frame can be expressed as

$$
t_{\text {cool }}^{\prime} \lesssim 10^{5} E_{\gamma, \mathrm{GeV}}^{-1 / 2} \xi^{3 / 4} \varkappa^{-3 / 4} z_{0,17}^{17 / 8} \mathrm{~s} .
$$

This timescale should be compared to the timescale characterizing the comoving blob acceleration time:

$$
t_{\mathrm{acc}}^{\prime} \sim 10^{5} z_{0,17}^{1 / 2} \mathrm{~s} .
$$

In Figure 2 (bottom left panel), we show the numerical computation of these timescales for the case of a conical jet. One can see that for the typical model parameters, these time scales are comparable. This result allows us to make three important conclusions about the considered scenario. Namely, (1) the fast cooling regime assumption should not be strongly violated in proton-synchrotron models for $\mathrm{GeV}$-emitting protons; (2) one should expect a cooling break in the high-energy domain; (3) the proton synchrotron mechanism appears to be an efficient radiation channel in the Fermi energy range. At lower energies, protons could remain uncooled, which may lead to requiring a hard particle distribution given the tight energetics of the flares in 3 C 454.3 plus additional observational constraints (see Section 4.2). It is often argued, based on the comparison of the proton cooling and jet dynamical time scales (see, e.g., Sikora 2011), that proton synchrotron is characterized by a very low efficiency as the gamma-ray production mechanism in AGN jets. However, as can be seen from Figure 2 (bottom left panel), proton synchrotron can produce a high radiation efficiency for the inferred jet properties.

The EIC channel is not strongly constrained in the JRGI scenario since the properties of the photon target are not well known and can vary within a broad range. Similar to the SSC case, the density and luminosity of the target photons can be estimated based on the ratio of the synchrotron and inverse Compton luminosities. This estimation yields the following luminosity:

$$
\begin{aligned}
L_{\mathrm{ph}, \mathrm{EIC}} & \sim 4 \pi z^{2} c w_{\mathrm{ph}} \\
& >3 \times 10^{47} f \varkappa \xi^{-1} z_{0,17}^{-1.5} \mathrm{erg} \mathrm{s}^{-1} .
\end{aligned}
$$

The value of this luminosity as a function of $D$ is shown in Figure 2 (bottom right panel). It is seen that the photon field luminosity required for the EIC scenario to work appears to be very high (accounting for $f \sim 100^{10}$ ), exceeding the reported luminosity of the broad-line region (BLR) unless $\varkappa$ is very small or the interaction region is located far enough from the central $\mathrm{BH}\left(z \gg 10^{17} \mathrm{~cm}\right)$.

There is another constraint related to the process of gammaray absorption through pair creation in the local radiation fields. The peaking energy of the radiation component detected with Fermi/LAT should be close to $100 \mathrm{MeV}$. Otherwise, the lower energy spectrum should be very hard not to violate the X-ray flux detected with Swift (Abdo et al. 2011). In the emitter comoving system, this would correspond to

$$
E_{\mathrm{ph}}^{\prime}=10 \Gamma_{\mathrm{b}, 1}^{-1} \mathrm{MeV},
$$

i.e., relatively close to the electron-positron creation threshold. Assuming a photon spectrum $\propto E^{-1.5}$ below the peak (it may be even harder; see Section 4.2), it is possible to compute the luminosity transferred to the secondary pairs:

$$
L_{\mathrm{sec}} \sim 5 \times 10^{48} L_{\gamma, 50}^{2} \Gamma_{\mathrm{b}, 1}^{-4} r_{\mathrm{b}, 15}^{-1} \mathrm{erg} \mathrm{s}^{-1},
$$

where $r_{\mathrm{b}, 15}=r_{b} / 10^{15} \mathrm{~cm}$ is the normalized blob radius. Substituting here the solutions obtained for the jet parameters (Equations (20)-(23)) in the limit of small $D$, one finds:

$$
L_{\mathrm{sec}} \sim 10^{49} z_{0,17}^{-2.25} \mathrm{erg} \mathrm{s}^{-1} .
$$

\footnotetext{
10 A similar consideration to the one done in the context of SSC applies here
} regarding EIC within the cloud, i.e., where potentially $x \ll 1$. 
This energy should be emitted either via synchrotron or inverse Compton radiation. The obtained estimate of the proper magnetic field from Equation (35) allows one to estimate the peaking energy for the synchrotron channel to be

$$
\hbar \omega_{\mathrm{sec}, \mathrm{sy}} \sim 10^{-2} \xi^{-1 / 2} \varkappa^{1 / 2} z_{0,17}^{-7 / 4} \mathrm{eV} .
$$

This component can be constrained by the infrared flux detected during the flare (Jorstad et al. 2012). If the dominant channel is inverse Compton scattering, the peak energy can be estimated as:

$$
\hbar \omega_{\mathrm{sec}, \mathrm{ic}} \sim 4\left(\frac{\epsilon_{\mathrm{EIC}}}{40 \mathrm{eV}}\right) \mathrm{MeV},
$$

where $\epsilon_{\mathrm{EIC}}$ is the target photon energy. Although this component would peak above the X-ray energy band, the lower energy tail may provide an important contribution to the reported flux level. Also, it is important to note that the inverse Compton photons may serve as targets for the absorption of primary gamma rays leading to a nonlinear regime of the emission formation. This effect, in particular, may make a self-consistent treatment of the EIC scenario very complex, and thus we leave this possibility for future dedicated studies. Nevertheless, we will account for internal gamma-ray absorption in the blob's own synchrotron field and we will comment on radiation reprocessing outside the blob in Section 4.2.

Despite being not very detailed, and order of magnitude, the above analysis shows that explaining the 2010 November flare detected from 3C454.3 in the framework of the JRGI scenario requires a jet with a very high ram pressure. Consequently, a magnetic field with a strength not far from equipartition, say a factor of 10 below, appears to be too strong for radiation processes other than synchrotron. On the other hand, leptonic synchrotron emission requires an extremely efficient acceleration process with an acceleration parameter $\eta<2$, which cannot be discarded but is perhaps unrealistic. Thus, proton synchrotron emission seems the most comfortable radiation channel given the restrictions imposed by the data and the JRGI scenario. In what follows, we test this process using a simple radiation model.

\subsection{Modeling the Lightcurve and the Spectrum}

To check whether the JRGI scenario plus synchrotron radiation can explain the observations in the case of magnetically dominated jets (i.e., $\varkappa=1$ ), we have computed the lightcurve of the 2010 November flare and the SED for one of its sub-flares. The radiation output was assumed to be dominated by proton synchrotron; EIC and SSC were neglected due to the strong magnetic field (see Section 4.1).

To derive the lightcurve, Equation (11) was used (see the determination of $F_{e}$ in Appendix B). In Figure 3, a computed lightcurve that approximately mimics the 2010 November flare is presented. The lightcurve has been obtained assuming four sub-flares of total (apparent) energy $10^{55} \mathrm{erg}$ each, plus a plateau component with a luminosity of $2 \times 10^{49} \mathrm{erg} \mathrm{s}^{-1}$. For each subflare, we have adopted $D=0.1$. The normalization of the lightcurve was determined adopting the following values: the Lorentz factor $\Gamma_{0}=28$, the ram pressure $P_{\mathrm{j}}=3 \times 10^{6} \mathrm{erg} \mathrm{cm}^{-3}$, blob radius $r_{\mathrm{b}}=2.7 \times 10^{15} \mathrm{~cm}$, and $\xi=0.3$. These parameters imply a minimum jet luminosity of $L_{\mathrm{j}}=2.3 \times 10^{48} \mathrm{erg} \mathrm{s}^{-1}$. The remaining parameters for the emitter are $z_{0}=1.3 \times 10^{17} \mathrm{~cm}$, and $M_{\mathrm{b}}=1.3 \times 10^{30} \mathrm{~g}$. The corresponding mass of the matter lost by the RG to explain the four sub-flares is $5 \times 10^{30} \mathrm{~g}$, not far from the upper limit given in Equation (2).

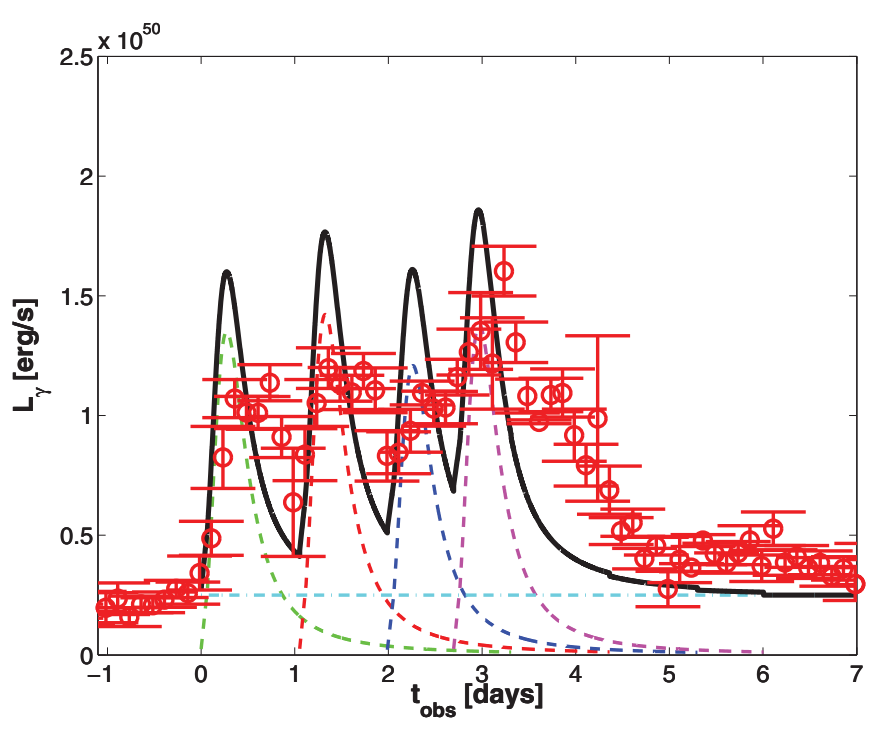

Figure 3. Lightcurve computed adopting the parameters $L_{\mathrm{j}}=2.3 \times 10^{48} \mathrm{erg} \mathrm{s}^{-1}$, $z=1.33 \times 10^{17} \mathrm{~cm}, \Gamma_{\mathrm{j}}=28, M_{\mathrm{c}}=1.3 \times 10^{30} \mathrm{~g}, r_{\mathrm{c}}=2.7 \times 10^{15} \mathrm{~cm}$, and $\xi=0.3$. We show four sub-flares (dashed lines), the plateau background (dotdashed line), and the sum of all of them (solid line). The observational data points and error bars are obtained from the Fermi/LAT $3 \mathrm{hr}$ binned count rates and photon index using a luminosity distance of $D_{\mathrm{L}}=5.5 \mathrm{Gpc}$ and assuming a pure power law spectrum between 0.1 and $5 \mathrm{GeV}$.

(A color version of this figure is available in the online journal.)

To calculate the SED, we adopted a spectrum for the injected protons $Q \propto E^{-p} \exp \left(-E / E_{\text {cut }}\right.$ ) and an homogeneous (onezone) emitter moving toward the observer with Lorentz factor $\Gamma_{\mathrm{b}}=12$. The minimum proton Lorentz factor was taken to be equal to the shock Lorentz factor in the blob frame, i.e., $E_{\text {min }}=\Gamma_{0} / \Gamma_{\mathrm{b}} m_{\mathrm{p}} c^{2}$. The cutoff energy, $E_{\text {cut }}$, was obtained by fixing $\eta=4 \times 10^{3}$ (see Equation (40) for details), i.e., a modest acceleration efficiency. For the maximum proton energy, i.e., how far beyond the cutoff the proton energy is considered, we adopted two values: $E_{\max }=\infty$ and $E_{\max }=3 E_{\text {cut }}$. Regarding the latter case, we note that assuming a sharp high-energy cut is very natural. The injection spectrum was selected to be hard, $p=1$, to optimize the required energetics. Interestingly, magnetic reconnection, in particular in magnetized jet-cloud interactions, has been postulated to provide hard particle spectra (see, e.g., Bosch-Ramon 2012 and references therein). The main motivation for a hard proton distribution is to avoid the need of a VHE budget, although the obtained synchrotron spectrum is also consistent with the observed IR and X-ray fluxes, as noted in the next paragraph. Significantly steeper particle distributions would enhance the required jet power by a factor of a few, but could also violate the observational constraints.

In Figure 4, the SED of a sub-flare is shown. The impact of the internal absorption on the gamma-ray spectrum is negligible, although the emission of the secondary pairs appear in the energy band constrained by optical measurements (Jorstad et al. 2012). For the chosen model parameters, the synchrotron secondary component goes right through the optical observational constraints, and for slightly higher $z_{0}$ values, the secondary emission will be well below the optical points (see Equation (48)). Also, we note that the obtained spectrum does not violate the X-ray upper limits obtained by Swift.

To illustrate the impact of external $\gamma \gamma$-absorption, we have introduced a photon field peaking at $40 \mathrm{eV}$ with a luminosity of $4 \times 10^{46} \mathrm{erg} \mathrm{s}^{-1}$, produced in a ring of radius $10^{18} \mathrm{~cm}$ 


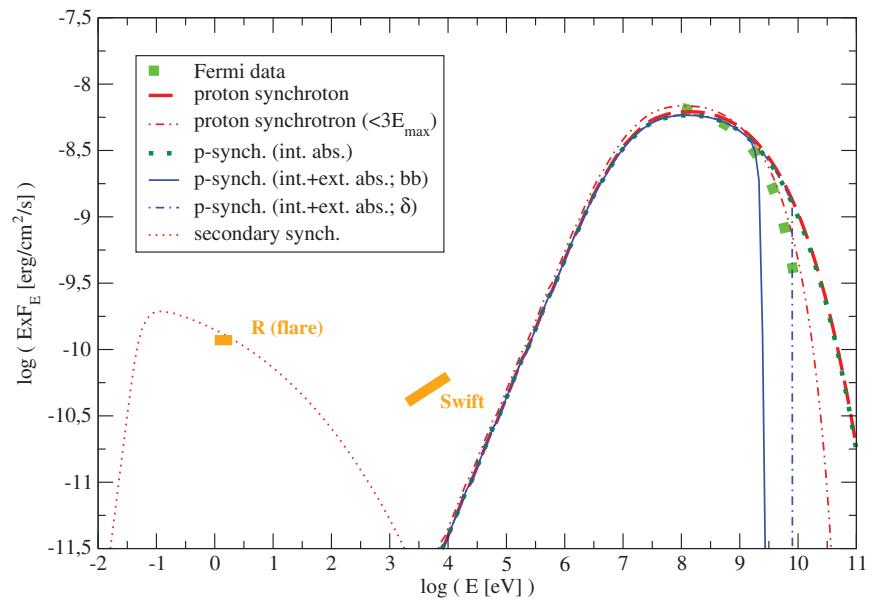

Figure 4. Computed SED of the synchrotron emission for a sub-flare of 2010 November. The thick dashed line shows the intrinsic gamma-ray emission for the case of $E_{\max }=\infty$. Dotted and dot-dot-dashed lines show the gamma-ray spectra corrected for internal absorption only for $E_{\max }=\infty$ and $E_{\max }=3 E_{\text {cut }}$, respectively. The thin solid and the dot-dashed lines correspond to the cases when absorption is dominated by a blackbody and a monoenergetic photon field, respectively. The computed synchrotron SED of the secondary pairs produced via internal pair creation is also shown (dotted line). The parameters of the flare are the same as in Figure 3. The observational data shown are from Fermi/LAT, Swift (Abdo et al. 2011), and the flux in the $R$ band (Jorstad et al. 2012).

(A color version of this figure is available in the online journal.)

at $z=0$ around the jet base. Two photon fields have been adopted, a blackbody and one represented by a $\delta$-function, to simulate the impact of a dominant spectroscopic line. As seen in Figure 4, the impact of such an external field can be important. The treatment of the secondary emission of the produced pairs is beyond the scope of this work. However, we note that the ambient magnetic field energy density could be well below that of the radiation, allowing electromagnetic cascades to develop, effectively increasing the gamma-ray transparency. In fact, if it were not the case, the secondary synchrotron emission would likely violate the constraints from the Swift data (see, e.g., Aharonian et al. 2008; Sitarek \& Bednarek 2010; Zacharopoulou et al. 2011). Extragalactic background light absorption is not relevant in the energy range of interest.

As seen in Figure 4, the spectral breaks induced by external absorption are very sharp and do not fit the Fermi/LAT data points. However, the properties of the external photon field can vary in a quite broad range, and it is expected that for some feasible photon field one could achieve a very good agreement between the emission spectrum and Fermi/LAT measurements (see, e.g., Stern \& Poutanen 2011). Instead of searching for such a field configuration, here we consider an alternative possibility. As mentioned above, we assume that the injection spectrum has not only an exponential cutoff at $E_{\text {cut }}$, but also a sharp upper limit at $E_{\max }=3 E_{\text {cut }}$. As seen in Figure 4, this assumption, naturally accounting for the fact that particles cannot have arbitrarily high energies in the source, allows one to qualitatively model the Fermi/LAT observations without invoking additional assumptions regarding external absorption.

In addition to optical photons, radio emission was also detected at the flare epoch and thought to be linked to the gamma-ray activity (Jorstad et al. 2012). This radiation is strongly sensitive to the details of the flow dynamics, and at this stage we will not try to interpret the radio observations. However, we note that the energetics involved in gamma-ray production are very large, and the JRGI scenario includes complex MHD and radiative processes, so it could easily accommodate the presence of a population of radio-emitting electrons at $z \geqslant z_{\text {flare }}$.

Swift X-rays could be also linked to the JRGI activity. As stated in Section 4.1, X-rays may come from secondary pairs produced via pair creation, or from a primary population of electrons(/positrons). However, as with the radio data, given the complexity of the problem we have not tried at this stage to explain the X-ray emission contemporaneous with the $\mathrm{GeV}$ flare.

\section{DISCUSSION}

Observations in the VHE domain put severe constraints on the size of the gamma-ray production site, supporting the picture of a very compact emitter. The concept of a blob as a compact emitter inside jets is often used to interpret the nonthermal emission of AGNs. This paradigm is supported by a broad range of observations, in particular bright knots moving downstream the jets. The origin of such blobs is not clear. It was suggested that the blobs form when an external object (e.g., a star or a cloud; see Araudo et al. 2010; Barkov et al. 2010 and references therein) penetrates the jet and loses its atmosphere due to the jet impact. Because of the much larger obstacle density and the velocity difference, the intruding matter cannot immediately dilute within the jet but has to be first heated and accelerated, which in supersonic jets will lead to strong shocks that can accelerate particles. Therefore, this scenario naturally presents specific characteristics that are hard to introduce in pure jet models. For instance, the large blob density makes possible the formation of a radiation component typically suppressed in AGNs, i.e., emission generated at proton-proton collisions (suggested to be behind the $\mathrm{TeV}$ emission detected from M87 by HESS; Barkov et al. 2012b). Moreover, since the size of the blobs produced in this scenario is not constrained by the gravitational radius of the central $\mathrm{BH}$, it was proposed that this process could be responsible for the very rapidly variable TeV emission detected from PKS 2155-304 (Barkov et al. 2012a; Aharonian et al. 2007). The JRGI scenario may have quite general applications, but the properties of the emission expected within its framework are quite strictly constrained. In particular, the available energy budget and expected lightcurve shape can be determined with a simple dynamical model, which describes the evolution of the blob in the jet. Depending on the jet properties and other factors like the viewing angle, the dominant emission channel can vary. However, the majority of the physical conclusions that can be inferred in the JRGI scenario are quite general because they concern the dynamics of compact blobs in relativistic jets, independent of the origin of the former and the nature of the radiation channel.

The observations of 3C454.3 with Fermi revealed several quite puzzling features, in particular the peculiar lightcurve, with a nearly steady plateau phase that was interrupted by an exceptionally bright flare. The detected flux corresponds to an apparent luminosity of $2 \times 10^{50} \mathrm{erg} \mathrm{s}^{-1}$, which almost unavoidably implies a presence of a very powerful jet (see, e.g., Bonnoli et al. 2011). In the case of powerful jets, the JRGI scenario should proceed in a quite specific way as compared to other cases already considered in the literature (Barkov et al. 2012a). In particular, the mass of the material initially removed from the star might be very large, resulting in rather long cloud expansion and acceleration timescales, the main flare being significantly delayed with respect to the moment of the star's entrance into the jet. The plateau emission would otherwise start 
just after the jet penetration, and come from the jet crushing the lighter clouds ejected from the stellar surface while the star travels through the jet. The duration of the plateau phase would be determined by the time required by the main cloud to expand and accelerate.

We have studied the lightcurve obtained with Fermi in the context of the JRGI scenario, aiming to reproduce three main properties of the flare: total energy, maximum luminosity and duration of the plateau stage. It was shown that the key properties of the jet, i.e., the jet ram pressure (linked to its luminosity) and Lorentz factor, as well as the cloud/blob characteristics, i.e., mass and cross-section, can be reconstructed as functions of the dimensionless parameter $D$. It was also shown that in the limit of small $D$ values, the parameter space is less demanding concerning the jet luminosity, and the key characteristics of the model saturate at values independent of $D$, which allows conclusive cross-checks of the scenario. In particular, the flare rise time appeared to be an independent parameter, with its value of $5 \mathrm{hr}$ closely matching the rising time of $4.5 \mathrm{hr}$ obtained observationally. Furthermore, it was shown (see Section 3.2) that for the inferred jet properties, the jet-induced stellar wind can provide a mass-loss rate large enough to generate a steady emission component with a luminosity comparable to that of the plateau.

Although the analysis of different radiation channels involves additional assumptions regarding the spectrum of the nonthermal particles and the density of the target fields, it was possible to show that for magnetic fields not far below equipartition (as expected in a magnetically launched jet; see Appendix A for details), all the conventional radiation channels can be discarded and the emission detected with Fermi can be produced through proton synchrotron emission (unless $\eta \rightarrow 1$, making electron synchrotron also feasible). We note that in this case, the emission from pairs created within the blob may also explain the reported optical enhancement at the flare epoch.

If the studied scenario is behind the 2010 November flare from 3C454.3, it has some important implications on the properties of AGN jets. On one hand, the obtained solution implies a superEddington jet. Although this requirement may appear somewhat extreme, given the exceptional properties of the source it cannot be ruled out (and in fact super-Eddington jets might be a rather common phenomenon; López-Corredoira \& Perucho 2012). In addition, the obtained solution (Equations (20)-(23)) implies a lower limit on the central $\mathrm{BH}$ mass that, for a location of the interaction region at $z_{0} \sim 10^{17} \mathrm{~cm}$, is roughly consistent with the measured values. Also, adopting a magnetically launched jet model, it is possible to infer the mass of the central $\mathrm{BH}$ as $M_{\mathrm{BH}, 9}=0.5 z_{0,17}^{1 / 2} \Gamma_{\mathrm{j}, 1.5}^{-1}$, which is again consistent with the measurements.

At the initial stage of the cloud acceleration, the intensity of the jet-cloud interaction is the highest, but the associated emission is not Doppler boosted and therefore very hard to detect in the high-energy regime. Nevertheless, there are other manifestations of the JRGI scenario that may be observed. In particular, as reported by León-Tavares et al. (2013), a significant enhancement of the line emission from the source was recorded during the flare epoch. The detected flux was $\sim 2 \times 10^{45} \mathrm{erg} \mathrm{s}^{-1}$, implying an ionizing luminosity at a level of $10^{46} \mathrm{erg} \mathrm{s}^{-1}$. According to our estimates, the initial blob acceleration period should be characterized by luminosities of $\sim 2 \times 10^{48} \mathrm{erg} \mathrm{s}^{-1}$, so only $1 \%$ of this luminosity would be required to explain the ionization line component. With the acceleration of the blob, its radiation gets beamed along the jet, thus reducing its impact on the BLR and thereby weakening any related line emission.

Since the duration of the expansion phase determines the delay between the onset of the plateau phase and the flare itself, it is important to check whether the suggested scenario is consistent with other flares registered with Fermi from the source, e.g., in 2009 December and 2010 April (Striani et al. 2010; Ackermann et al. 2010). This issue can be addressed through a simple scaling that relates the duration of the plateau phase to the total energy released during the active phase: $t_{\mathrm{pl}} \propto E_{\mathrm{tot}}^{1 / 2}$ (assuming a steady jet, one can obtain from Equations (C3) and (C7) that $P_{0} \times \Gamma_{0}^{3}=$ const, which yields in the scaling dependence). Therefore, for the previous events, with energy releases 1-2 orders of magnitude smaller than that of the 2010 November flare, a rough estimation of the plateau duration gives plateau durations between 1.3 and 4 days, consistent with observations.

The rate of the RG penetrating the jet deserves some discussion. This rate is determined by the stellar density in the close vicinity of the central $\mathrm{BH}$, say at distances of $\sim 1$ pc. Conventionally, one adopts a power law dependence of the RG density on distance of the sort: $\rho_{*}=\rho_{0}(z / 1 \mathrm{pc})^{-a}$. Given that the stellar velocity can be estimated as $V_{*} \approx 600 M_{\mathrm{BH}, 8}^{1 / 2} r_{\mathrm{pc}}^{-1 / 2} \mathrm{~km} \mathrm{~s}^{-1}$, one can estimate the stellar penetration rate for the distance interval $(z, z+d z)$ :

$$
d \dot{M}_{*} \simeq \rho_{*} V_{*} z \Gamma_{\mathrm{j}}^{-1} d z,
$$

where we have used $z / \Gamma_{\mathrm{j}}$ as the jet radius.

In the scenario proposed here, the $\mathrm{RG}$ penetration takes place at a typical height of $10^{17} \mathrm{~cm}$. We can therefore roughly derive the rate of these events by integrating Equation (51) over the $z$ interval $\approx(0.3-3) \times 10^{17} \mathrm{~cm}:$

$$
\dot{M}_{*} \simeq 6 \times 10^{-4} \frac{\rho_{0} M_{\mathrm{BH}, 8}^{1 / 2}}{\Gamma_{\mathrm{j}}} \int_{0.01}^{0.1} x^{1 / 2-a} d x\left(\mathrm{pc}^{3} \mathrm{yr}^{-1}\right)
$$

where $x=z / 1 \mathrm{pc}$ is a dimensionless radius. A rather broad range of values of the $a$-parameter is discussed in the literature (Bisnovatyi-Kogan et al. 1982; Murphy et al. 1991; Merritt \& Szell 2006). In particular, Murphy et al. (1991) obtained $a=7 / 4$ for small stellar densities $\left(\sim 10^{6} M_{\odot} \mathrm{pc}^{-3}\right)$ and $a=0.5$ for high densities $\left(\sim 10^{8} M_{\odot} \mathrm{pc}^{-3}\right)$. The value of the parameter $\rho_{0}$ can be estimated through the mass of the stellar cusp around the central BH: $\rho_{0}=(1-a / 3) \bar{\rho}_{*}$, where $\bar{\rho}_{*} \simeq 10^{6-8} M_{\odot} \mathrm{pc}^{-3}$ is the averaged (typical) stellar density for $z<1$ pc.

The integral term in Equation (52) spans approximately two orders of magnitude, between 0.1 and 15 , for the given range of $a$. We therefore conclude that based on the results obtained by Murphy et al. (1991), one should expect a rather weak dependence of Equation (52) on the value of $a$. As a reference, we take the high stellar density case with $a=0.5$, obtaining:

$$
\dot{M}_{*} \simeq 6 \times 10^{3} \frac{M_{\mathrm{BH}, 8}^{1 / 2} M_{\odot}}{\Gamma_{\mathrm{j}}} \mathrm{yr}^{-1} .
$$

Given the typical jet Lorentz factor of $\sim 30$ and a $\sim 1 \%$ fraction of $\mathrm{RG}$ stars, the above equation corresponds to $\sim 2$ events $\mathrm{yr}^{-1}$ for $M_{\mathrm{BH}, 8}=1$, which is consistent with observations.

Our study (and before, Barkov et al. 2012a) shows that the penetration of a star into the base of the jet in a powerful blazar leads to a very characteristic dynamical evolution. In fact, the JRGI scenario has quite-constrained physics with basically only 
one free parameter: the nonthermal efficiency. The specific conditions in the jet/blob interaction region will determine, as long as particle acceleration occurs, the dominant radiation process, but in any case powerful high-emission seems natural in the proposed scenario.

The authors are thankful to S. Kelner for useful discussions and Benoit Lott for providing observational data. The research leading to these results has received funding from the European Union Seventh Framework Program (FP7/2007-2013) under grant agreement PIEF-GA-2009-252463. V.B.-R. acknowledges support by the Spanish Ministerio de Ciencia e Innovación (MICINN) under grants AYA2010-21782-C03-01 and FPA2010-22056-C06-02. V.B.-R. acknowledges financial support from MINECO through a Ramón y Cajal fellowship. This research has been supported by the Marie Curie Career Integration Grant 321520. M.V.B. acknowledges partial support by RFBR grant 12-02-01336-a. A.V.D. was supported by an appointment at the NASA Goddard Space Flight Center, administered by CRESST/UMD through a contract with NASA, and by grants from the NASA Astrophysics Theory Program 10-ATP10-0171.

\section{APPENDIX A}

\section{THE STRUCTURE OF MAGNETICALLY DRIVEN JETS}

Although the process of jet formation is not fully understood, recent hydrodynamical studies by different groups have shown that the BZ process may be at work in AGNs. These studies suggest that, once formed, jets may be magnetically accelerated. In this scenario, the jet base is expected to be magnetically dominated at $z \leqslant 1$ pc (Komissarov et al. 2007; Barkov \& Komissarov 2008; Beskin 2010).

During jet propagation, the magnetic field energy can be transformed into bulk kinetic energy, with a simple prescription for the bulk Lorentz factor at the linear acceleration stage of the form (Beskin \& Nokhrina 2006):

$$
\Gamma_{\mathrm{j}} \approx \frac{\omega}{4 r_{\mathrm{g}}}
$$

where $\omega$ and $r_{\mathrm{g}}$ are the jet and the $\mathrm{BH}$ gravitational radius, respectively. The opening angle of the jet is expected to be $\theta=\omega / z \approx 1 / \Gamma_{\mathrm{j}}$ (Komissarov et al. 2009). This leads to few useful relations between different jet parameters:

$$
\Gamma_{\mathrm{j}}^{2} \approx \frac{z}{4 r_{\mathrm{g}}}, \quad \theta^{2} z \approx \frac{\omega^{2}}{z} \approx \frac{z}{\Gamma_{\mathrm{j}}^{2}} \approx 4 r_{\mathrm{g}}
$$

It is also useful to express the above relation in the units used all through the paper:

$$
\begin{gathered}
\Gamma_{\mathrm{j}, 1.5}^{2}=0.17\left(=0.4^{2}\right) z_{0,17} M_{\mathrm{BH}, 9}^{-1}, \\
\theta_{-1}^{2} z_{0,17}=0.6 M_{\mathrm{BH}, 9} .
\end{gathered}
$$

The jet magnetic field in the comoving frame can also be derived:

$$
B_{\mathrm{c}} \approx \frac{2}{z}\left(\frac{L_{\mathrm{j}}}{c}\right)^{1 / 2} \approx 120 z_{0,17}^{-1} L_{j, 48}^{1 / 2} \mathrm{G}
$$

\section{APPENDIX B \\ ON BLOB LUMINOSITY IN THE CASE OF HEAVY BLOBS}

If the mass of the blob is high, implying a low value of the $D$-parameter, then blobs can travel a significant distance along the jet. Therefore, the jet properties can change, and this should have an impact on the emission associated with the blobs. These effects can be estimated via the approach suggested by Barkov et al. (2012a). The dynamics of the blob are characterized by:

$$
\frac{d \Gamma_{\mathrm{b}}}{d t}=\left(\Gamma_{\mathrm{b}}^{-2}-\Gamma_{\mathrm{b}}^{2} \Gamma_{\mathrm{j}}^{-4}\right) \frac{P_{\mathrm{j}} \pi r_{\mathrm{b}}^{2}}{4 c M_{\mathrm{b}}},
$$

where $P_{\mathrm{j}}$ is the ram pressure of the jet. Since the emission of the blob is important only when it moves relativistically, it is safe to take $z=z_{0}+c t$ and the initial condition $\left.\Gamma_{\mathrm{b}}\right|_{t=0}=1$. The above equation can be modified to a dimensionless form:

$$
\frac{d g}{d y}=\left(\frac{\left(\Gamma_{\mathrm{j}} / \Gamma_{0}\right)^{2}}{g^{2}}-\frac{g^{2}}{\left(\Gamma_{j} / \Gamma_{0}\right)^{2}}\right) \frac{D}{\left(\Gamma_{j} / \Gamma_{0}\right)^{2}\left(P_{0} / P_{\mathrm{j}}\right)},
$$

where $z=z_{0} y ; \omega_{0}, \Gamma_{0}$ and $P_{0}$ are the jet radius, ram pressure and Lorentz factor at $z=z_{0}$; respectively, $g=\Gamma_{\mathrm{b}} / \Gamma_{0}$; and the dimensionless parameter $D$ is determined as

$$
D=\frac{P_{0} \pi r_{\mathrm{b}}^{2} z_{0}}{4 c^{2} M_{\mathrm{b}} \Gamma_{0}^{3}}
$$

This approach is nearly identical to the one developed earlier, but here one accounts for the possible change of the properties of the jet (e.g., for the increase in the jet Lorentz factor).

If $D \gg 1$, the blob gets rapidly involved into the jet motion, so the solution is identical to the one obtained in Barkov et al. (2012a) even if the properties of the jet are changing with $z$. However, if $D<1$, the structure of the jet may have some influence on the properties of the emission. In particular, for purely magnetically driven jets, i.e., with a parabolic shape, the above equation can be simplified, since the following relations hold:

$$
\begin{gathered}
\omega_{0}=2 \sqrt{r_{\mathrm{g}} z_{0}}, \\
\omega=\omega_{0} y^{1 / 2}, \quad P_{\mathrm{j}}=P_{0} y^{-1}, \\
\Gamma_{\mathrm{j}}=z_{0} / \omega_{0} y^{1 / 2}=\Gamma_{0} y^{1 / 2} .
\end{gathered}
$$

Thus, the equation of motion (B2) yields

$$
\frac{d g}{d y}=\left(\frac{y}{g^{2}}-\frac{g^{2}}{y}\right) \frac{D}{y^{2}},
$$

with the boundary condition $\left.g\right|_{y=1}=\omega_{0} / z_{0}$. Although this equation does not have any analytical solution, an asymptotic solution can be derived for $D \ll 1$ (i.e., the jet structure is important):

$$
g_{\text {ap }}=\left[\left(\omega_{0} / z_{0}\right)^{3}+3 D \ln y\right]^{1 / 3} .
$$

For the case of a conical jet (i.e., $\Gamma_{\mathrm{j}}=$ const, $\omega=z / \Gamma_{\mathrm{j}}$ and $P_{\mathrm{j}}=P_{0} y^{-2}$ ), the equation of motion reduces to the equation:

$$
\frac{d g}{d y}=\left(g^{-2}-g^{2}\right) \frac{D}{y^{2}}
$$



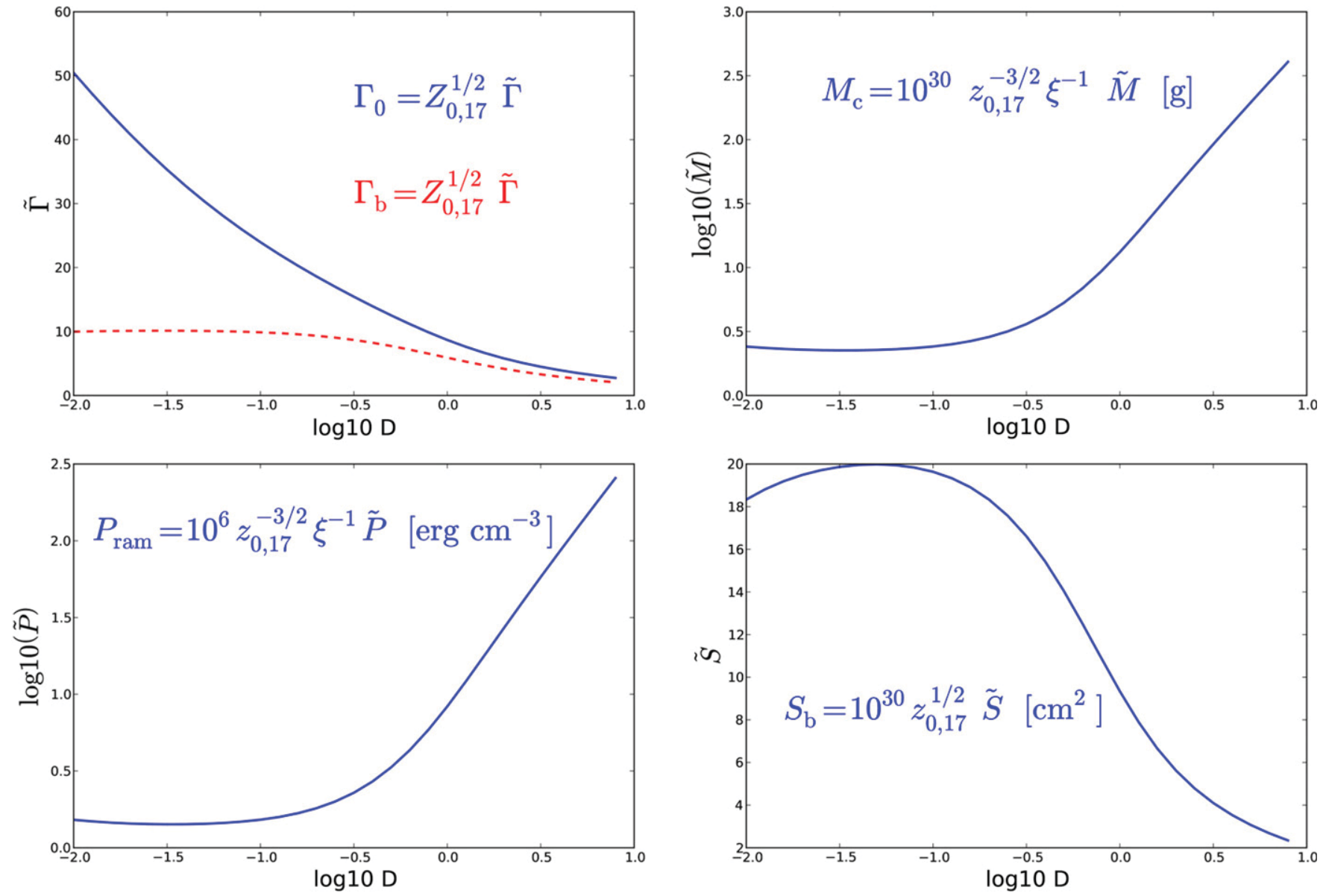

Figure 5. The derived jet parameters as a function of the $D$-parameter.

(A color version of this figure is available in the online journal.)

which allows an analytical solution (for details, see Barkov et al. 2012a). However, since the analytical solution is rather bulky, we use the asymptotic solution:

$$
g_{\mathrm{ac}}=\left[\left(\omega_{0} / z_{0}\right)^{3}+3 D(y-1) / y\right]^{1 / 3},
$$

which is valid for $D \ll 1$. Since the models of parabolic and conical jets correspond to the most feasible jet model, we will consider the realization of the JRGI scenario for these two cases. The obtained asymptotic solutions allow one to clearly estimate the differences that depend on the specific jet configuration.

The intensity of the nonthermal emission of the blob, corrected for Doppler boosting, has the following form:

$$
L_{\gamma}=4 \xi L_{\mathrm{j}}\left(\frac{r_{\mathrm{b}}}{\omega_{0}}\right)^{2} \Gamma_{0}^{2} F_{\mathrm{e}}(y)
$$

where $F_{\mathrm{e}}(y)=g^{4} /\left(P_{0} / P_{\mathrm{j}}\right)\left(1 / g^{2}-g^{2} /\left(\Gamma_{\mathrm{j}} / \Gamma_{0}\right)^{4}\right)$ is the correction function.

The maximum value of the correction function, $F_{\mathrm{e}, \max }$, depends on the value of the $D$-parameter and on the structure of the jet. If $D \geqslant 1$, it saturates at a constant value of $F_{\mathrm{e}, \max } \approx 0.4$ independent of the jet properties. In the case of $D \ll 1$, the asymptotic solutions show that the maximum of the correction function is reached at $y_{\mathrm{m}} \approx 1.95$, with $F_{\mathrm{e}, \max } \approx 0.82 D^{2 / 3}$ for a parabolic jet, and for a conical jet, $y_{\mathrm{m}} \approx 1.33$ and $F_{\mathrm{e}, \max } \approx 0.46 D^{2 / 3}$. In the intermediate range, $0.1<D<1$, $F_{\mathrm{e} \text {,max }}$ is $\sim 0.5 D^{0.5}$ and $\sim 0.3 D^{0.5}$ for the parabolic and conical jets, respectively. The location of the maximum $y_{\mathrm{m}}$ has a weak dependence on $D$ in this regime; i.e., depending on the structure of the jet, the maximum intensity, $F_{\mathrm{e}, \max }$, can vary by a factor of $\sim 2$ in the case of $D<1$.

The total energy emitted by a blob can be obtained via the integration of the luminosity over the observer time $(d \tau=$ $\left.z_{0} d y /\left(2 c \Gamma_{\mathrm{b}}^{2}\right)\right)$ :

$$
E_{\gamma} \simeq 8 \xi\left(\int_{1}^{\infty} d y F_{\mathrm{e}} \frac{D}{g^{2}}\right) M_{\mathrm{b} / \mathrm{c}} c^{2} \Gamma_{\mathrm{j}}^{3}=8 \xi \bar{F}_{\mathrm{e}} M_{\mathrm{b} / \mathrm{c}} c^{2} \Gamma_{\mathrm{j}}^{3} .
$$

This equation defines the function $\bar{F}_{\mathrm{e}}$.

For large values of the $D$-parameter, the integration term in the above equation can be obtained analytically if one accounts for the relation $F_{\mathrm{e}}=(d g / d y) g^{4} / D$ :

$$
\bar{F}_{\mathrm{e}}=\int_{1}^{\infty} d y F_{\mathrm{e}} \frac{D}{g^{2}}=\int_{0}^{g_{\max }} d g g^{2}=\frac{1}{3} g_{\max }^{3}
$$

Here we note that, in the case of an accelerating jet, the integral determining the total emitted energy is divergent. Formally, this is so because $g_{\max } \rightarrow \infty$. Therefore, the integration should be artificially truncated at some point. Physically, this point would correspond to the moment when the blob gets homogenized in the jet. For large values of $D$, the blob speed rapidly reaches the jet velocity, and one should adopt $g_{\max }=1$. For small $D$ values, 
we use $\int_{1}^{\infty} d y F_{\mathrm{e}}\left(D / g^{2}\right)=D$, which is a precise identity for the case of a conical jet, and corresponds to a truncation at $y \sim 3$ for a parabolic jet. Therefore, for the present order-of-magnitude estimates, one can estimate the energy emitted by the blob as

$$
\bar{F}_{\mathrm{e}}=\int_{1}^{\infty} d y F_{\mathrm{e}} \frac{D}{g^{2}}=\min \left(D, \frac{1}{3}\right) .
$$

Since the structure of the jet only imposes uncertainties on the order of two, for the order-of-magnitude estimates presented in this paper we use the solution obtained for the conical jet structure. To use the solution obtained for the parabolic structure of the jet, one should properly describe the process of the blob homogenization in the jet, which is beyond the scope of this work.

\section{APPENDIX C}

\section{A SOLUTION FOR THE JET PARAMETERS}

One can obtain a simplified analytical solution which allows the determination of the jet parameters. Ignoring the weak dependence on $R_{*}$ in Equation (17), one obtains:

$$
\begin{aligned}
M_{\mathrm{c}, 30}= & \frac{3 \times 10^{-2}}{\xi F_{\mathrm{e}, \max } \Gamma_{\mathrm{j}, 1.5}^{2} S_{\mathrm{b}, 32}}\left(\frac{L_{\gamma}}{2 \times 10^{50} \mathrm{erg}}\right) \\
& \times\left(\frac{t_{\mathrm{pl}}}{13 \text { days }}\right)^{-2}\left(\frac{1+z_{\mathrm{rs}}}{1.859}\right)^{2} .
\end{aligned}
$$

From Equation (15), one can infer the mass of the cloud:

$$
M_{\mathrm{c}, 30}=\frac{0.1}{\xi \bar{F}_{\mathrm{e}} \Gamma_{\mathrm{j}, 1.5}^{3}}\left(\frac{E_{\gamma}}{2 \times 10^{55} \mathrm{erg}}\right),
$$

and Equation (14) can be re-normalized as

$$
P_{0}=2 \times 10^{4} \xi^{-1} F_{\mathrm{e}, \max }^{-1} \Gamma_{0,1.5}^{-2} S_{\mathrm{b}, 32}^{-1}\left(\frac{L_{\gamma}}{2 \times 10^{50} \mathrm{erg}}\right) \mathrm{erg} \mathrm{cm}^{-3}
$$

Finally, the definition of the $D$-parameter, Equation (6), provides the fourth required equation to determine the parameters $P_{0}, S_{\mathrm{b}}$, $M_{\mathrm{c}}$ and $\Gamma_{0}$. Here, we assume that the flare was the result of the superposition of four sub-flares (i.e., $M_{\mathrm{c}}=4 M_{\mathrm{b}}$ ):

$$
D=\frac{6 \times 10^{-3} z_{0,17}}{F_{\mathrm{e}, \max } \xi M_{\mathrm{c}, 30} \Gamma_{0,1.5}^{5}}\left(\frac{L_{\gamma}}{2 \times 10^{50} \mathrm{erg}}\right) .
$$

Equations $(\mathrm{C} 1)-(\mathrm{C} 4)$ have a unique solution, which characterizes the parameters $P_{0}, S_{\mathrm{b}}, M_{\mathrm{c}}$ and $\Gamma_{0}$ as functions of $D$ :

$$
\begin{aligned}
P_{0}= & \times 10^{6} \frac{F_{\mathrm{e}, \max }^{1.5} D^{1.5}}{\bar{F}_{\mathrm{e}}^{2.5 \xi} \xi z_{0,17}^{1.5}}\left(\frac{L_{\gamma}}{2 \times 10^{50} \mathrm{erg}}\right)^{-1.5}\left(\frac{t_{\mathrm{pl}}}{13 \text { days }}\right)^{-2} \\
& \times\left(\frac{E_{\gamma}}{2 \times 10^{55} \mathrm{erg}}\right)^{2.5} \mathrm{erg} \mathrm{cm}^{-3} \\
M_{\mathrm{c}}= & 4 M_{\mathrm{b}}=5 \times 10^{30} \frac{F_{\mathrm{e}, \max }^{1.5} D^{1.5}}{\bar{F}_{\mathrm{e}}^{2.5} \xi z_{0,17}^{1.5}}\left(\frac{L_{\gamma}}{2 \times 10^{50} \mathrm{erg}}\right)^{-1.5} \\
& \times\left(\frac{E_{\gamma}}{2 \times 10^{55} \mathrm{erg}}\right)^{2.5} \mathrm{~g},
\end{aligned}
$$

$$
\Gamma_{0}=8\left(\frac{\bar{F}_{\mathrm{e}} z_{0,17}}{F_{\mathrm{e}, \max } D}\right)^{0.5}\left(\frac{L_{\gamma}}{2 \times 10^{50 \mathrm{erg}}}\right)^{0.5}\left(\frac{E_{\gamma}}{2 \times 10^{55 \mathrm{erg}}}\right)^{-0.5},
$$

and

$$
\begin{aligned}
S_{\mathrm{b}}= & 8 \times 10^{30} \frac{z_{0,17}^{0.5} \bar{F}_{\mathrm{e}}^{1.5}}{F_{\mathrm{e}, \max }^{1.5} D^{0.5}}\left(\frac{L_{\gamma}}{2 \times 10^{50} \mathrm{erg}}\right)^{1.5}\left(\frac{t_{\mathrm{pl}}}{13 \text { days }}\right)^{2} \\
& \times\left(\frac{E_{\gamma}}{2 \times 10^{55 \mathrm{erg}}}\right)^{-1.5} \mathrm{~cm}^{2} .
\end{aligned}
$$

The values $F_{\mathrm{e}, \max }$ and $\bar{F}_{\mathrm{e}}$ depend on the value of the $D$ parameter and the structure of the jet. To illustrate the feasibility of the derived solution, in Figure 5 we show the jet parameters as functions of the $D$-parameter.

\section{REFERENCES}

Abdo, A. A., Ackermann, M., Ajello, M., et al. 2011, ApJL, 733, L26 Ackermann, M., Ajello, M., Baldini, L., et al. 2010, ApJ, 721, 1383

Aharonian, F., Akhperjanian, A. G., Bazer-Bachi, A. R., et al. 2007, ApJL, 664, L71

Aharonian, F. A. 2000, NewA, 5, 377

Aharonian, F. A., Belyanin, A. A., Derishev, E. V., Kocharovsky, V. V., \& Kocharovsky, V. V. 2002, PhRvD, 66, 023005

Aharonian, F. A., Khangulyan, D., \& Costamante, L. 2008, MNRAS, 387, 1206 Araudo, A. T., Bosch-Ramon, V., \& Romero, G. E. 2010, A\&A, 522, A97

Barkov, M. V., Aharonian, F. A., Bogovalov, S. V., Kelner, S. R., \& Khangulyan, D. 2012a, ApJ, 749, 119

Barkov, M. V., Aharonian, F. A., \& Bosch-Ramon, V. 2010, ApJ, 724, 1517

Barkov, M. V., Bosch-Ramon, V., \& Aharonian, F. A. 2012b, ApJ, 755, 170

Barkov, M. V., \& Komissarov, S. S. 2008, IJMPD, 17, 1669

Basko, M. M., \& Sunyaev, R. A. 1973, Ap\&SS, 23, 117

Basko, M. M., Sunyaev, R. A., Hatchett, S., \& McCray, R. 1977, ApJ, 215, 276

Beskin, V. S. 2010, PhyU, 180, 1241

Beskin, V. S., \& Nokhrina, E. E. 2006, MNRAS, 367, 375

Bisnovatyi-Kogan, G. S., Churaev, R. S., \& Kolosov, B. I. 1982, A\&A, 113, 179

Blandford, R. D., \& Königl, A. 1979, ApJ, 232, 34

Blandford, R. D., \& Znajek, R. L. 1977, MNRAS, 179, 433

Bonnoli, G., Ghisellini, G., Foschini, L., Tavecchio, F., \& Ghirlanda, G. 2011, MNRAS, 410, 368

Bosch-Ramon, V. 2012, A\&A, 542, A125

Bosch-Ramon, V., Perucho, M., \& Barkov, M. V. 2012, A\&A, 539, A69

Dorodnitsyn, A., Kallman, T., \& Proga, D. 2008a, ApJL, 675, L5

Dorodnitsyn, A., Kallman, T., \& Proga, D. 2008b, ApJ, 687, 97

Ghisellini, G., Foschini, L., Tavecchio, F., \& Pian, E. 2007, MNRAS, 382, L82

Gregori, G., Miniati, F., Ryu, D., \& Jones, T. W. 2000, ApJ, 543, 775

Gu, M., Cao, X., \& Jiang, D. R. 2001, MNRAS, 327, 1111

Imshennik, V. S. 1972, SPhD, 17, 576

Jorstad, S. G., Marscher, A. P., Joshi, M., et al. 2012, arXiv:1205.0520

Jorstad, S. G., Marscher, A. P., Larionov, V. M., et al. 2010, ApJ, 715, 362

Katarzyński, K., \& Ghisellini, G. 2007, A\&A, 463, 529

Kelner, S. R., Aharonian, F. A., \& Khangulyan, D. 2013, ApJ, in press (arXiv:1304.0493)

Komissarov, S. S., Barkov, M. V., Vlahakis, N., \& Königl, A. 2007, MNRAS, 380, 51

Komissarov, S. S., Vlahakis, N., Königl, A., \& Barkov, M. V. 2009, MNRAS, 394, 1182

Lemoine, M., \& Waxman, E. 2009, JCAP, 11, 9

León-Tavares, J., Chavushyan, V., Patiño-Álvarez, V., et al. 2013, ApJL, 763, L36

López-Corredoira, M., \& Perucho, M. 2012, A\&A, 544, A56

Lovelace, R. V. E. 1976, Natur, 262, 649

Merritt, D., \& Szell, A. 2006, ApJ, 648, 890

Mücke, A., \& Protheroe, R. J. 2001, APh, 15, 121

Murphy, B. W., Cohn, H. N., \& Durisen, R. H. 1991, ApJ, 370, 60

Nakamura, F., McKee, C. F., Klein, R. I., \& Fisher, R. T. 2006, ApJS, 164,477

Pittard, J. M., Hartquist, T. W., \& Falle, S. A. E. G. 2010, MNRAS, 405, 821

Ruffini, R., \& Wilson, J. R. 1975, PhRvD, 12, 2959 
Sikora, M. 2011, in IAU Symp. 275, Jets at All Scales, ed. G. E. Romero, R. A. Sunyaev, \& T. Belloni (Cambridge: Cambridge Univ. Press), 59

Sikora, M., Moderski, R., \& Madejski, G. M. 2008, ApJ, 675,71

Sitarek, J., \& Bednarek, W. 2010, MNRAS, 409, 662

Stern, B. E., \& Poutanen, J. 2011, MNRAS, 417, L11
Striani, E., Vercellone, S., Tavani, M., et al. 2010, ApJ, 718, 455

Tchekhovskoy, A., Narayan, R., \& McKinney, J. C. 2010, NewA, 15, 749

Vercellone, S., Striani, E., Vittorini, V., et al. 2011, ApJL, 736, L38

Wehrle, A. E., Marscher, A. P., Jorstad, S. G., et al. 2012, ApJ, 758, 72

Zacharopoulou, O., Khangulyan, D., Aharonian, F. A., \& Costamante, L. 2011, ApJ, 738, 157 